\title{
Engineering the grain boundary network of thin films via ion-irradiation: Towards improved electromigration resistance
}

\author{
Huan Ma ${ }^{\mathrm{a}}$, Fabio Lamattina ${ }^{\mathrm{b}}$, Ivan Shorubalko ${ }^{\mathrm{b}}$, Ralph Spolenak ${ }^{\mathrm{a}}$, Matteo Seita ${ }^{\mathrm{c}, *}$ \\ ${ }^{a}$ Laboratory for Nanometallurgy, Department of Materials, ETH Zurich, Vladimir-Prelog-Weg 5, 8093 Zurich, Switzerland \\ ${ }^{b}$ Laboratory for Reliability Science and Technology, EMPA, 8600 Dübendorf, Switzerland \\ ${ }^{c}$ Department of Materials Science and Engineering, Massachusetts Institute of Technology, MA 02139, USA
}

\begin{abstract}
Controlling the grain boundary network of small-scale materials-such as coatings and thin films - is an ambitious goal that would ultimately enable the production of components with tailored properties and improved reliability. In this work we present a new techniquewhich we term ion-induced grain boundary engineering (iGBE) — to engineer the character distribution and connectivity of grain boundaries in gold films in situ, as they are being deposited. iGBE consists of a repeated sequence of ion-induced material removal and material deposition which results in the selection and growth of crystal grains in twinned relationship. This phenomenon yields a substantial increase in the density and connectivity of $\Sigma 3$ twin boundaries, which have renowned beneficial effect on the material's resistance to intergranular degradation. We confirm the improved reliability of iGBE microstructures by assessing their resistance to electromigration - one of the most common causes of failure in integrated circuit interconnects. We find that, through iGBE, interconnect lifetime increases by three orders of magnitude at standard operating conditions. Since iGBE is materialinsensitive and compatible with standard microfabrication technology, we expect it to have significant impact on microelectronics industry.
\end{abstract}

Keywords: Grain boundary engineering; Thin films; Ion-irradiation; Microstructure design; Electromigration

\footnotetext{
*Correspondence should be addressed to Matteo Seita (E-mail: matteos@mit.edu; Tel.: 00-1-617-258-8038).
} 


\section{Introduction}

Most engineering materials are polycrystalline: they consist of an aggregate of differently oriented crystal grains joined along grain boundaries (GBs) [1]. Although they typically comprise a small fraction of the material's volume, GBs have tremendous impact on its properties, including strength and ductility [2-4], diffusion [5-7], resistance to environmentassisted failure $[8,9]$ and radiation tolerance $[10,11]$. Indeed, by engineering the distribution and connectivity of GBs - namely the GB network - such properties can be improved by many orders of magnitude [12-15]. In metals and metal alloys, the GB distribution can be tailored by processing raw materials through specific thermo-mechanical treatments-a sequence of cold work and anneal—which take the name of grain boundary engineering (GBE) [16-20]. GBE yields an increased proportion of "special" GBs in the microstructureprimarily $\Sigma 3$ boundaries — which have the effect of improving the material's performance and its resistance to intergranular degradation [21].

While GBE is largely employed for the fabrication of high-performance bulk materials, its use at the small-scale — such as on coatings or thin films — is still limited, due to the challenge of selectively deform and anneal small volumes of material [22]. Here we present a new method to engineer the GB network of as-deposited films and thus improve their performance. Our method consists of a combination of ion-irradiation and material deposition processes to selectively control the crystal grain orientation distribution, in situ, during sputter-deposition. We demonstrate the method-which we refer to as ion-induced grain boundary engineering (iGBE) — using gold (Au) films and we prove the beneficial effect of iGBE by assessing the material's resistance to electromigration (EM) - a GB-controlled degradation phenomenon which causes failure of interconnects in integrated circuits [23]. 


\section{Experimental}

$\mathrm{Au}$ deposition is performed by DC magnetron sputtering (PVD Products, Inc.) at room temperature on (100) silicon wafers coated with $50 \mathrm{~nm}$ of $\mathrm{SiO}_{2}$ and $50 \mathrm{~nm}$ of $\mathrm{Si}_{3} \mathrm{~N}_{4}$. To improve the adhesion of the film to the wafer, a $10 \mathrm{~nm}$ thick Ti layer is deposited prior to Au. The base pressure in the sputter chamber is better than $10^{-6}$ mbar. During deposition, the pressure is increased to $10^{-3}$ mbar by flowing pure argon (Ar) in the chamber and the silicon substrate is kept in rotation (with a speed of $12 \mathrm{rpm}$ ) in order to obtain a homogeneous film thickness. Ion-irradiation is carried out by means of a gridded ion-source (KRI KDC 40) with collimated and neutralized $\mathrm{Ar}^{+}$beam. The ion-source is installed directly in the sputter tool at a fixed angle of $35^{\circ}$ with respect to the film surface normal. This configuration allows for in situ ion-irradiation of the deposited films. The ion-energy, ion-current, and irradiation time are kept constant throughout the experiments presented here at $1.2 \mathrm{keV}, 5 \mathrm{~mA}$, and 8 minutes, respectively. Upon irradiation, the base pressure in the chamber is increased to $\sim 4 \times 10^{-4}$ mbar and the substrate rotation is turned off.

The film microstructure is characterized by means of a high-resolution scanning electron microscope (SEM, Magellan 400 FEI) using both secondary electron (SE) and backscattered electron (BSE) detectors. Grain orientation information is assessed via electron backscatter diffraction (EBSD) using a FEI Quanta 200 FEG SEM. Only EBSD data with confidence index (CI) larger than 0.1 is considered. Quantities such as crystallographic texture spread, average grain size, and area fraction of specific grain orientations are measured from EBSD data using the OIM Analysis Software (EDAX, Inc). The GB character distribution and the triple junction (TJ) distribution are measured from cleaned EBSD data using MTex 3.4.1 [24] - a free software for the MATLAB environment. The cleanup procedure used is similar to others found in the literature [25-27] and consists of grain CI standardization, neighbor orientation correlation with level 1 , and a single iteration of grain dilation using grain 
tolerance angle of $5^{\circ}$ and minimum grain size of 2 pixels. GBs are classified according to the coincidence site lattice model [28], using Brandon's criterion [29, 30]. The film grain structure is characterized along the cross-section using high-resolution SEM, transmission electron microscopy (TEM, FEI Tecnai F30 and FEI Talos F200X). The TEM analysis is carried out in both bright field (BF) and high-angle annular dark-field (HAADF) modes. Cross-sections and TEM lamellae are prepared using a focused ion beam (FIB) microscope (NVision 40, Carl Zeiss).

EM tests are carried out using a four-point probe method [31] on arrays of Au lines, patterned from $500 \mathrm{~nm}$ thick films via lithography. The lines are $10 \mu \mathrm{m}$ wide and $700 \mu \mathrm{m}$ long. All lines are coated with a $200 \mathrm{~nm} \mathrm{SiN}_{\mathrm{x}}$ layer by plasma enhanced chemical vapor deposition (Plasmalab 80 Plus, Oxford Instruments) at $300^{\circ} \mathrm{C}$. EM tests are performed in a shielded wafer prober at controlled temperature, ranging from 125 to $200^{\circ} \mathrm{C}$. The current density used for all tests is $1.3 \times 10^{7} \mathrm{~A} / \mathrm{cm}^{2}$. All lines are tested until open circuit failure. 


\section{Microstructure control via iGBE}

Fig. 1 illustrates the elements of iGBE via schematics as well as EBSD maps taken from representative samples. First, a $50 \mathrm{~nm}$ thick Au film — hereafter referred to as the referenceis sputter-deposited onto a substrate. The reference microstructure consists of columnar grains with an average size of $\sim 60 \mathrm{~nm}$ and strong (111) fiber texture normal to the film surface. The fiber texture - a common feature in the microstructure of thin FCC films [32]is clearly visible from the (111) pole figure in Fig. 1a, where the central peak and the continuous ring at $\sim 70^{\circ}$ indicate that all grains have the same surface normal, but random inplane orientation, respectively.

After deposition, the reference film is irradiated at a fixed angle of $35^{\circ}$ with respect to the surface normal (see schematics in Fig. 1b). As the energetic ions strike the film surface, two different phenomena take place in the crystal grains, depending on their orientation: ionmilling and ion-channeling. Grains that are randomly-oriented with respect to the ion-beam direction undergo ion-milling and thus are progressively removed from the sample surface [33]. Conversely, those oriented such that their open-most crystal-axis is parallel to the ionbeam undergo ion-channeling and thus survive ion-irradiation [34]. We refer to the combination of both phenomena as selective grain milling (SGM). The EBSD measurement of the reference after SGM is reported in Fig. 1b. Notably, the (111) pole figure now exhibits three symmetric peaks at $\sim 70^{\circ}$ from the normal, which suggests that all grains left on the surface have similar crystallographic orientation $[35,36]$.

In FCC materials, such as $\mathrm{Au}$, the easiest channeling-axes coincide with the $<110>$ crystallographic directions [37]. Owing to the pre-existing (111) out-of-plane texture in the reference film, all grains in the microstructure exhibit three possible $<110>$ channeling axes at an angle of $35^{\circ}$ from the surface normal — exactly the angle at which the ion-beam is set. However, since the reference film is held steady during SGM, only one specific subset of 
grains is found in channeling-orientation and thus survives SGM. These are the grains appearing in the EBSD map in Fig. 1b. The orientation spread of the surviving grains is $\sim 20^{\circ}$, as measured by EBSD. This value is in good agreement with the maximum angular deviation from the nominal channeling-axis that is allowed for the ion-energy, ion-species, and target material used in this study. In fact, following the model proposed by Lindhard [34], we compute a maximum deviation of:

$\psi_{c}=\left(\frac{3 a^{2} Z_{a} Z_{b} e^{2}}{E_{i} d^{3}}\right)^{1 / 4}=21^{\circ}$

where $Z_{a}$ and $Z_{b}$ are the atomic numbers of ions and target atoms, respectively, $e^{2}=14.4 \mathrm{eV}$ $\AA$ (the square of the elementary charge), $E_{i}$ the ion energy $(1.2 \mathrm{keV}), d$ the distance between two atoms along the $<110>$ channel wall in $\mathrm{Au}(2.9 \AA)$, and $a=a_{B} 0.8853\left(Z_{a}^{2 / 3}+Z_{b}^{2 / 3}\right)^{-1 / 2}$, where $a_{B}$ is the Bohr radius. The agreement between the measured orientation spread and $\psi_{c}$ confirms that the grains remaining on the sample surface after SGM are in channelingorientation and that all the other randomly-oriented grains were selectively removed. In our experiments, 8 minutes is the shortest irradiation time that yields complete removal of randomly-oriented grains. In fact, after 8 minutes the material's coverage on the sample surface is measured to be $25.3 \%$, which is very close to the measured fraction of channelingoriented grains $(25.7 \%$, see Table 1$)$ in the reference film and the predicted values in a fiber textured film $(27.5 \%$, see reference [35]).

In the next step of iGBE, a supplemental $50 \mathrm{~nm} \mathrm{Au}$ film is sputter-deposited onto the irradiated reference without breaking the vacuum. The resulting microstructure- - hereafter referred to as $1 \times i G B E$ - exhibits a higher fraction of channeling-oriented grains $(\sim 39.7 \%$, see Table 1) as well as a large proportion of twinned grains $(\sim 26.4 \%$, see Table 1$)$. This can be seen from the pole figure in Fig. 1c, where the six-fold symmetry about the (111) fiber texture suggests the presence of two twinned orientations rotated by $60^{\circ}$. The increased 
fraction of channeling-oriented grains is due to the epitaxial growth of the pre-existing channeling-oriented grains - namely those which survived the first SGM [38]. At the same time, the favorable conditions for twin nucleation onto (111) crystal planes during physical vapor deposition of FCC materials leads to the preferential growth of grains in twinned orientation $[39,40]$. This means that upon the supplemental deposition of $50 \mathrm{~nm}$ of $\mathrm{Au}$, the adatoms diffusing to the channeling-oriented grains can arrange themselves in two-equally favorable - crystallographic orientations: one which follows the parent orientation (namely the channeling-orientation), and one that is in a twinned relationship to them, which results into the nucleation of a new grain. However, not all new grains fall in the latter category. In fact $\sim 6.3 \%$ of the grains in $1 \times \mathrm{iGBE}$ exhibits no crystallographic relationship to the channeling-orientation, besides displaying the same (111) fiber texture (see Table 1). These are the grains that nucleated in areas that were depleted of material after SGM. Because we only consider EBSD data with $\mathrm{CI}>0.1$, a large fraction of material is not assigned a crystallographic orientation and appears in the EBSD scans in black color. We believe that the low CI in these regions is due to the small grain size of the films, which approaches the resolution limit of EBSD. As a result, during the EBSD scan it is often the case that multiple diffraction patterns from different grains are superposed and not recognized by the EBSD software. Such a shortcoming leads to uncertainties in the evaluation of the area fraction of different grains.

Fig. 1e shows the EBSD map of a Au film subjected to two cycles of repeated SGM and deposition of $50 \mathrm{~nm} \mathrm{Au}$ supplement-hereafter referred to as $2 \times i G B E$. The resulting film comprises $\sim 48.7 \%$ of channeling-oriented grains and $\sim 22.2 \%$ of twinned-oriented grains (see Table 1). This yields a microstructure that is predominantly bi-crystalline, since it is composed for $\sim 70.9 \%$ by only two crystallographic orientations in a twinned relationship (Fig. 1e). The origin of the bi-crystalline microstructure stems from the higher fraction of 
channeling-oriented grains that is induced through the first iGBE cycle. In fact, despite SGM removes all grains that are not in channeling-orientation-including the twinned ones - the higher fraction of channeling-oriented grains in $1 \times$ iGBE compared to the reference (from $25.7 \%$ to $39.7 \%$, see Table 1) ensures that fewer grains will nucleate with random crystallographic orientation upon the second Au supplement.

In Fig. 2 we show how Au coverage in $50 \mathrm{~nm}$ thick films changes during the entire iGBE process (as depicted in Fig. 1) by means of SE and BSE micrographs. Since the SE and BSE signals are function of surface morphology and material's atomic number, respectively, the information carried by the corresponding micrographs is complementary and can be used to reliably assess film integrity. From Fig. 2a,c,e, it is clear that all films after both initial and supplemental deposition are continuous. Conversely, after SGM, the films are removed with exception of the channeling-oriented grains (in bright contrast against the dark contrast from the substrate), which survive the ion-milling process (see Fig. $2 b$ and d). The area fractions of the channeling-oriented grains from the SE and BSE analysis are $\sim 23.9 \%$ and $\sim 46.1 \%$ after the first (Fig. 2b) and second (Fig. 2d) SGM step, respectively. These values are in good agreement with those measured by EBSD in Fig. $1 \mathrm{~b}$ and $d \quad(\sim 25.3 \%$ and $\sim 44.9 \%$, respectively).

The GB type that joins two twinned grains is called $\Sigma 3$ boundary [28]. $\Sigma 3$ boundaries are the foremost type of boundaries among the "special" GBs which are purposefully introduced in the microstructure during conventional GBE [41]. The large proportion of twinned-grains induced through the iGBE cycles leads to a progressive increase in the number fraction of $\Sigma 3$ boundaries: from $15.7 \%$ (in the reference) to $17.5 \%$ (in $1 \times \mathrm{iGBE}$ ) to $18.4 \%$ (in $2 \times \mathrm{iGBE}$ ), as can be seen from the GB character distribution reported in Fig. 3. This means that after only two cycles of iGBE the total number fraction of $\Sigma 3$ boundaries can be increased by $17 \%$. Beside $\Sigma 3$ boundaries, other "special" GBs are also engineered through iGBE (see Fig. 3). 
engineer the GB character distribution in thin metal films, iGBE also allows engineering GB connectivity by promoting "special GBs" to connect.

Because of the large fraction of material that cannot be indexed by EBSD (see Fig. 1a,c,e and Table 1), we note that the GB character and TJ distributions presented in Fig. 3 and Fig. 4, respectively, contain uncertainties. As a result, rather than focusing on the absolute number fraction of specific GB types (such as $\Sigma 3$ boundaries) or TJs (such as TJ2 and TJ3), we highlight their relative change through iGBE. 


\section{Templated growth of iGBE films}

All the different samples-reference, $1 \times \mathrm{iGBE}$, and $2 \times \mathrm{iGBE}$ - can be used as "microstructure templates" to grow thicker films that inherit the same texture and grain structure. This is accomplished by depositing an additional $450 \mathrm{~nm}$ of material on each microstructure template without breaking the vacuum. High-resolution SEM images from the cross-section of the newly produced films (now $500 \mathrm{~nm}$ thick) confirm that most grains are columnar and that the GBs extend through the entire film thickness (see Fig. 5). The EBSD orientation maps and corresponding (111) pole figures for all $500 \mathrm{~nm}$ thick films are presented in Fig. 6a. The grain orientation distribution in $500 \mathrm{~nm}$ thick films is reported in Table 1 for comparison with that in the microstructure templates. In general, we find that the thicker films exhibit a bi-crystalline microstructure similar to that of microstructure templates, with strong out-of-plane and in-plane textures. This finding confirms that thin iGBE films can be used as microstructure templates to grow thicker films with controlled microstructure. Some differences can be found in the average grain size and grain orientation distribution when comparing $50 \mathrm{~nm}$ to $500 \mathrm{~nm}$ thick films. In fact, thicker films comprise grains that are about double the size of those in microstructure templates (the average grain size increases from $\sim 60 \mathrm{~nm}$ to $\sim 120 \mathrm{~nm}$ ). Moreover, upon thickening we measure a larger fraction of twinned-oriented grains and a decreased fraction of randomly-oriented grains. Both the increase in average grain size and the change in grain orientation distribution may be attributed to competitive grain growth upon sputter-deposition [32]. During competitive grain growth, grains with orientations that are more energetically favorable (e.g. for FCC materials, those with surface normal parallel to the $<111>$ direction), or grains with larger diameter grow faster than others [32]. Since during sputter-deposition of FCC metal films, such as Au, grains are known to grow even at room temperature [44], we expect competitive grain growth to occur in all our samples upon deposition of the additional $450 \mathrm{~nm}$. All grains in our films 
have surface normal parallel to the $<111>$ direction and thus are, presumably, equally favorable to grow. However, some grains in iGBE films (i.e. $1 \times$ iGBE and $2 \times$ iGBE) have different size, depending on their in-plane orientation. In fact we can classify all grains into three groups based on their in-plane orientation with respect to the incident ion beam direction during SGM: channeling-, twinned- and randomly-oriented grains. Upon film thickening, channeling-oriented grains grow larger because they survive SGM (as we explained in Section 3). When a twinned-oriented grain nucleates over a channeling-oriented grain during supplemental deposition, presumably it will inherit the same (large) size. Moreover, the probability of twin nucleation increases with increasing grain size [27]. Conversely, all randomly-oriented grains nucleate in areas that are depleted of material. As a result, randomly-oriented grains are smaller and, therefore, they will be gradually overgrown by the channeling- and twinned-oriented grains during competitive grain growth. Following these arguments, upon film thickening we expect a decrease in the fraction of randomlyoriented grains while an increase in that of the twinned-oriented grains, which is indeed the trend reported in Table 1. However, the observed differences in grain orientation distribution may also be partially attributed to the large fraction of material that cannot be indexed by EBSD in $50 \mathrm{~nm}$ thick films (see Fig. 1a,c,e and Table 1), which may introduce uncertainties in the measured area fraction of the different grain families.

Because of their columnar grain structure (see Fig. 5), we treat all the $500 \mathrm{~nm}$ thick films as 2-D microstructures, whose GB character distribution and network can be directly characterized at the film surface by EBSD. Fig. 6b shows that the fraction of $\Sigma 1$ and $\Sigma 3$ boundaries in $500 \mathrm{~nm}$ thick films progressively increases through the iGBE cycles. This trend is comparable to what we find in the microstructure templates (see Fig. 3). However, the absolute number fractions are significantly different than those in the microstructure templates, especially in the iGBE samples. The fraction of $\Sigma 3$ boundaries reaches $16.1 \%$ in 
reference (compared to $15.7 \%$ in the microstructure templates), $23.6 \%$ in $1 \times$ iGBE (compared to $17.5 \%$ ) and $28.2 \%$ in $2 \times$ iGBE (compared to $18.4 \%$ ). This means that after only two cycles of iGBE on the microstructure template, a thicker film can be grown with $75 \%$ more $\Sigma 3$ boundaries than what it would naturally comprise. This result is justified by the higher fraction of twinned-oriented grains in $500 \mathrm{~nm}$ thick films (see Table 1). We may speculate that the decrease in $\Sigma 1$ boundaries from $44.5 \%$ to $40 \%$ upon film thickening of the $2 \times$ iGBE sample is the result of the increased probability for channeling-oriented grains to turn into twinned-oriented ones for the reasons outlined in the foregoing. As a result, fewer channeling-oriented grains will be adjacent.

The analysis of the TJ distributions in $500 \mathrm{~nm}$ thick films is reported in Fig. 7. When compared to microstructure templates (Fig. 4), $500 \mathrm{~nm}$ thick films show similar trends in the evolution of GB network topology. TJ0's and TJ1's decrease, while TJ2's and TJ3's increase up to $38.1 \%$ and $31 \%$, respectively, in just two iGBE cycles. As we will show in Section 5 and 6 , this change has profound effects on the properties of iGBE materials. 


\section{Electromigration testing of different microstructures}

To prove that iGBE is an effective strategy to enhance the performance of small-scale materials, we assess the EM resistance of all three microstructures using accelerated EM tests. EM consists in the progressive deterioration of interconnects in microelectronic circuits, when they carry high current densities [23]. Over time, the electrons drive the atoms of the crystalline material to diffuse across the interconnect line from cathode to anode. This atomic migration results in the formation of voids (at the cathode side) and hillocks or whiskers (at the anode side), which ultimately result into the failure of the interconnect (i.e. in the form of an open or short circuit) [23]. Since atomic diffusion is faster along GBs than across the crystal lattice [45], materials' resistance to EM damage is function of their GB character distribution and connectivity—namely the GB network [46].

We perform EM testing on arrays of interconnect lines which we patterned from the 500 $\mathrm{nm}$ thick reference, $1 \times \mathrm{iGBE}$, and $2 \times$ iGBE films by lithography (see schematics in Fig. $8 \mathrm{a}$ ). We design the interconnects to be $\sim 80$ grains wide to ensure that there are no size-effects to affect the line's EM behavior, such as when the line width is comparable to the average grain size (i.e. "bamboo-like microstructures") [47]. Since Au does not form a stable surface oxide layer, we coat the lines with $200 \mathrm{~nm}$ of $\operatorname{SiN}_{\mathrm{x}}$ to prevent atomic surface diffusion from dominating EM damage [23]. Because we see no grain growth in the lines for temperatures as high as $400^{\circ} \mathrm{C}$ (see Fig. 9) - nor we see changes in the grain structure of the tested lines (see Fig. 8c-e) - we conclude that the crystallographic texture, GB character and TJ distribution do not evolve during EM testing. For each of the microstructures produced, we test from 3 to 5 lines at each test-temperature considered. Each line is tested to open circuit failure and the corresponding time to failure $(T T F)$ is plotted versus the inverse of the test-temperature in the Arrhenius plot shown in Fig. 8b. To account for the Joule heating induced in the lines when the current is applied, the following procedure is routinely carried out. Before EM testing, the 
foregoing analysis, we conclude that the EM behavior of the tested microstructures is function only of the GB network.

To quantify EM resistance and compare the behavior of the different microstructures, we calculate the effective activation energy for EM, $E_{a}$, using Black's formula [49]:

$T T F=A j^{-n} \exp \left(\frac{E_{a}}{k_{B} T}\right)$

where $A$ is a pre-exponential factor, $k_{B}$ the Boltzmann constant, $j$ the current density, and $n$ the acceleration factor. Both reference and $1 \times \mathrm{iGBE}$ lines have similar activation energy of $\sim 0.55 \mathrm{eV}$, while $2 \times$ iGBE lines exhibit a much higher value of $\sim 1.22 \mathrm{eV}$ (see Fig. $8 \mathrm{~b}$ ). Differences in EM behavior are also noticeable in the lines' TTF. For instance the extrapolated $T T F$ of $2 \times \mathrm{iGBE}$ at $100^{\circ} \mathrm{C}$ - the typical operating temperature in integrated circuits [23] - is 2 and 3 orders of magnitude longer than that of $1 \times \mathrm{iGBE}$ and reference lines, respectively (see Fig. 8b). These findings demonstrate that iGBE allows for the production of interconnects with improved resistance to EM. Moreover, the drastic increase in $E_{a}$ after two iGBE cycles suggests a change in the kinetics of EM-induced damage. Indeed, the value of $0.55 \mathrm{eV}$ is comparable to what is reported in literature for GB-mediated $\mathrm{EM}$ in $\mathrm{Au}(0.42-$ $0.98 \mathrm{eV}$ ) [50-53]. Conversely, the higher value of $1.22 \mathrm{eV}$ in $2 \times \mathrm{iGBE}$ indicates slower atomic migration, approaching that of lattice diffusion $(\sim 1.8 \mathrm{eV})[54]$. 


\section{Discussion}

The change in GB character distribution and GB connectivity induced through iGBE may be at the origin of the measured difference in EM behavior of the interconnect lines-and thus of their improved resistance to EM. Indeed, both $\Sigma 1$ and $\Sigma 3$ boundaries are known to be low-energy interfaces and to exhibit low atomic diffusivity $[45,55,56]$. The highest number fraction of $\Sigma 1$ and $\Sigma 3$ boundaries obtained in $2 \times \mathrm{iGBE}$ and the tendency for such boundaries to be connected together (as shown from the TJ distribution analysis) could be enough to force atomic migration through low-diffusivity paths, yielding a measurable difference in $E_{a}$. In the following, we are going to use percolation theory to interpret our EM results and test this hypothesis. Following the work by Kononenko et al. [46] we consider only $\Sigma 1$ and $\Sigma 3$ to be low-diffusivity GBs and we compute their cumulative number fraction, $p$, in the different microstructures that we tested. We then define two percolation thresholds, $p_{H}$ and $p_{L}$, corresponding to having a connected path of high- and low-diffusivity GBs across the interconnect line, respectively. We use the two thresholds to study which microstructures comprise percolating network of GBs with similar diffusivity. If $p<p_{H}$, a connected path of high-diffusivity GBs must exist in the line, while if $p>p_{H}$ then no such path exists. Conversely, if $p>p_{L}$ a connected path of low-diffusivity GBs exists across the interconnect. The values of $p_{H}$ and $p_{L}$ are taken from the theoretical work by Frary and Schuh [57, 58], who calculated percolation thresholds in 2-D microstructures for both fiber textured and twinned microstructures. Indeed, because our films comprise columnar grains (see Fig. 5 and Fig. 8ce), they can be considered as 2-D microstructures. Moreover, since the iGBE microstructures are both textured and twinned, we define $p_{H}$ and $p_{L}$ as a range of values between the corresponding theoretical thresholds (reported in Table 3). The connectivity of high- and lowdiffusivity GBs for the different microstructures is reported in Fig. 11a. Our analysis indicates that none of the samples produced in this study comprises a percolating path of 
high-diffusivity GBs (i.e. all microstructures exhibit $p>p_{H}$ ). However, percolation of lowdiffusivity GBs is expected in $2 \times \mathrm{iGBE}$ — exactly the sample showing the highest $E_{a}$.

To interpret this result, we first correlate the measured EM behavior of our lines to their effective diffusivity, considering only GBs as the atomic migration pathway. Simulations of GB networks have shown that the effective diffusivity of a GB network is determined by the effective atomic flux across the network and, therefore, that it is sensitive to the percolation of high-diffusivity GBs [6]. Since $p>p_{H}$ for all the three microstructures we tested, we expect their effective diffusivities to be similar [6]. If the EM resistance of a line were function of the effective diffusivity of its microstructure, then we would expect all the three microstructures to exhibit similar EM behavior. That is clearly not the case. The activation energy for EM that we measured in $2 \times \mathrm{iGBE}$ lines is more than twice that of the reference and $1 \times$ iGBE lines. Moreover, the effective diffusivity of a microstructure does not depend on the percolation of low-diffusivity GBs. However our EM tests show that the microstructure with highest resistance to $\mathrm{EM}$ is $2 \times \mathrm{iGBE}$, which is the only one exhibiting a percolating network of low-diffusivity GBs.

We conclude that EM resistance must be evaluated on the basis of $p_{L}$, not $p_{H}$. The rationale to support our conclusion is that EM-induced damage forms at locations - or nodes - in the microstructure where there is a strong difference in the atomic migration flux — namely where the flux diverges. In our interconnect lines, flux divergence nodes are the interfaces between clusters of high- and low-diffusivity GBs [59]. Since both reference and 1×iGBE lines have no percolating network of GBs with similar diffusivity $\left(p_{H}<p<p_{L}\right)$, we expect them to consist of a series of high- and low-diffusivity GB clusters-and thus to contain flux divergence nodes from the cathode to the anode. The only type of lines that exhibit a connected path of GBs with similar (low) diffusivity across the entire interconnect is indeed $2 \times$ iGBE $\left(p>p_{L}\right)$. Based on this argument we can explain why $2 \times$ iGBE exhibits the highest 
resistance to EM damage (i.e. higher $E_{a}$ ). This concept is schematically illustrated in Fig. $11 \mathrm{~b}$, using an electric circuit model. Here, clusters of low- and high-diffusivity GBs are represented as low- and high-conductivity resistors, respectively. Nodes of large divergence in the atomic flux can be found when both types of resistors are connected in series across the interconnect line.

Using this simple model, we can speculate on the origin of the measured difference in TTF between the reference and $1 \times$ iGBE samples. Because of the higher number fraction of lowdiffusivity GBs in $1 \times$ iGBE, we can expect a lower density of flux divergence nodes across the lines. Intuitively, this translates into a slower degradation of the $1 \times \mathrm{iGBE}$ lines and thus into an improved lifetime, which is superior to that of the reference sample by a factor 2 across the entire range of test-temperatures (see Fig. 8b). However, because $p_{H}<p<p_{L}$ in both reference and $1 \times \mathrm{iGBE}$ microstructures, EM kinetics - and thus $E_{a}$ - is similar in both types of microstructures.

One interesting case is that of $p<p_{H}$ (see schematics in Fig. 11b), namely when a connected path of high-diffusivity GBs exists across the interconnect line. While we have no experimental assessment of the EM behavior of such a microstructure, based on the foregoing discussion it might be tempting to conclude that it should have high resistance to EM (owing to the lack of flux divergence nodes across an hypothetical interconnect). However, the presence of a high-diffusivity path across the line would make its use in microelectronic devices impracticable. In fact, any interface between the interconnect and other functional units or diffusion barriers in the device would turn into a flux divergence node [23]. Owing to the fast atomic diffusion across the percolating path of high-diffusivity GBs, the interconnect lifetime would thus be comparable to - if not shorter than — that of the reference-type lines.

Another important aspect to consider when comparing the EM behavior of the reference, $1 \times \mathrm{iGBE}$ and $2 \times \mathrm{iGBE}$ lines is that different processing conditions are used to produce the 
where $D^{\prime}$ and $D=D_{0} \exp \left(-Q / k_{B} T\right)$ refer to the atomic diffusivities with and without stresses, respectively. $D_{0}$ is a pre-exponential factor, $Q$ the activation energy for diffusion without stresses, $\Omega$ the atomic volume, $B$ an effective modulus, which depends on the passivation conditions as well as the material tested, and $\sigma$ the hydrostatic stress. $Q-\sigma \Omega$ represents the activation energy for diffusion considering the effect of stresses. Assuming that $Q$ is the same for all tested microstructures and that the measured difference in activation energy $(0.67 \mathrm{eV}=1.22-0.55 \mathrm{eV})$ stems solely from the effect of stresses, a stress of $3.4 \mathrm{GPa}$ would be required to explain the difference in the activation energy $(0.67 \mathrm{eV})$, according to Eq. (8). However, $3.4 \mathrm{GPa}$ is much greater than the yield strength of $\mathrm{Au}[66,67]$ and even the fracture strength of the passivation layer of $\operatorname{SiN}_{\mathrm{x}}$ [68]. Therefore it would be impossible for the metal lines to sustain such level of stress. Consequently, we conclude that the mentioned difference in activation energy cannot be attributed to the effect of stresses, rather to the change of the GB network in the microstructures.

Interestingly, the foregoing analysis suggests that our EM test—performed on nominally identical interconnect lines, except for their GB network-is capable of measuring GB diffusion in both high- and low-diffusivity GBs. In fact, while in reference and $1 \times \mathrm{iGBE}$ samples EM kinetics is governed by high-diffusivity GBs, in $2 \times \mathrm{iGBE}$ it must be a function of the atomic flux divergence between low-diffusivity GBs and the crystal grains (i.e. lattice diffusion). Therefore the measured value of $1.22 \mathrm{eV}$ must result from the average between $\Sigma 1$ and $\Sigma 3$ boundary self-diffusivities. On the other hand, the value of $0.55 \mathrm{eV}$ must be the average self-diffusivity of all other GB types in the microstructure. 


\section{Summary}

In summary, we have proved that iGBE is a novel method to engineer the GB network of thin metal films, in situ, to an unprecedented level of detail. iGBE is based on the repeated sequence of SGM and supplemental material deposition which enables the production of microstructures comprising controlled fraction of low-diffusivity GBs-specifically, $\Sigma 1$ and $\Sigma 3$ boundaries. We showed that iGBE markedly affects GB connectivity. By applying different iGBE cycles, it is in fact possible to produce films with tailored GB network topology, which show completely different behavior. To prove the beneficial effect of iGBE, we tested the EM resistance of three differently processed Au interconnects and found a progressive improvement in the lines' lifetime. By designing the EM tests to only probe the role of the GB network, we were able to explain the origin of the improved performance using percolation theory and to estimate atomic self-diffusivity of different GB types. Since iGBE is a material-insensitive method, it can be readily applied to a variety of different cubic materials - both FCC and BCC—with no requirements for additional treatments—such as annealings — or for specific substrates. Most importantly, the method is readily integrable in standard microfabrication technology for the production of interconnects with superior resistance to EM. 


\section{Acknowledgements}

The authors would like to thank Dr. K. Kunze and Dr. J. Reuteler (ScopeM, ETH Zurich) for their help with EBSD and FIB work, Dr. A. Sologubenko for her help with TEM characterization and analysis, and the FIRST clean room (ETH Zurich) for magnetron sputtering facility. The authors also acknowledge financial support by the Swiss National Science Foundation under Contract No. 200021_140532 and 200021_118021.

\section{References}

[1] A.P. Sutton, R.W. Balluffi. Interfaces in crystalline materials, Clarendon Press, Oxford, 1995.

[2] K. Lu, L. Lu, S. Suresh. Strengthening Materials by Engineering Coherent Internal Boundaries at the Nanoscale, Science 324 (2009) 349-352.

[3] L. Lu, X. Chen, X. Huang, K. Lu. Revealing the Maximum Strength in Nanotwinned Copper, Science 323 (2009) 607-610.

[4] Y.J. Wei, Y.Q. Li, L.C. Zhu, Y. Liu, X.Q. Lei, G. Wang, Y.X. Wu, Z.L. Mi, J.B. Liu, H.T. Wang, H.J. Gao. Evading the strength- ductility trade-off dilemma in steel through gradient hierarchical nanotwins, Nat. Commun. 5 (2014).

[5] A. Suzuki, Y. Mishin. Diffusion mechanisms in grain boundaries in conventional and nanostructured materials, Minerals, Metals \& Materials Soc, Warrendale, 2004.

[6] Y. Chen, C.A. Schuh. Diffusion on grain boundary networks: Percolation theory and effective medium approximations, Acta Mater. 54 (2006) 4709-4720.

[7] O.K. Johnson, L. Li, M.J. Demkowicz, C.A. Schuh. Inferring grain boundary structure-property relations from effective property measurements, J Mater Sci 50 (2015) 6907-6919. 
[8] E.M. Lehockey, D. Limoges, G. Palumbo, J. Sklarchuk, K. Tomantschger, A. Vincze. On improving the corrosion and growth resistance of positive $\mathrm{Pb}$-acid battery grids by grain boundary engineering, J. Power Sources 78 (1999) 79-83.

[9] M. Seita, J.P. Hanson, S. Gradecak, M.J. Demkowicz. The dual role of coherent twin boundaries in hydrogen embrittlement, Nature Communications 6 (2015).

[10] T.D. Shen, S. Feng, M. Tang, J.A. Valdez, Y. Wang, K.E. Sickafus. Enhanced radiation tolerance in nanocrystalline MgGa2O4, Appl. Phys. Lett. 90 (2007).

[11] B.P. Uberuaga, L.J. Vernon, E. Martinez, A.F. Voter. The relationship between grain boundary structure, defect mobility, and grain boundary sink efficiency, Sci Rep 5 (2015).

[12] E.M. Lehockey, G. Palumbo. On the creep behaviour of grain boundary engineered nickel, Mater. Sci. Eng. A-Struct. Mater. Prop. Microstruct. Process. 237 (1997) 168-172.

[13] E.M. Lehockey, G. Palumbo, P. Lin. Improving the weldability and service performance of nickel- and iron-based superalloys by grain boundary engineering, Metall. Mater. Trans. A-Phys. Metall. Mater. Sci. 29 (1998) 3069-3079.

[14] D.P. Norton, A. Goyal, J.D. Budai, D.K. Christen, D.M. Kroeger, E.D. Specht, Q. He, B. Saffian, M. Paranthaman, C.E. Klabunde, D.F. Lee, B.C. Sales, F.A. List. Epitaxial YBa2Cu3O7 on biaxially textured nickel (001): An approach to superconducting tapes with high critical current density, Science 274 (1996) 755-757.

[15] K. Indarajīta, W. Gust, L. Kozma. Handbook of grain and interphase boundary diffusion data, Ziegler, Stuttgart, 1989.

[16] T. Watanabe. An Approach to Grain-Boundary Design for Strong and Ductile Polycrystals, Res Mech 11 (1984) 47-84.

[17] V. Randle. Mechanism of twinning-induced grain boundary engineering in low stacking-fault energy materials, Acta Mater. 47 (1999) 4187-4196. 
[18] T. Watanabe, S. Tsurekawa. The control of brittleness and development of desirable mechanical properties in polycrystalline systems by grain boundary engineering, Acta Mater 47 (1999) 4171-4185.

[19] S. Tsurekawa, S. Nakamichi, T. Watanabe. Correlation of grain boundary connectivity with grain boundary character distribution in austenitic stainless steel, Acta Mater 54 (2006) 3617-3626.

[20] G. Palumbo, P.J. King, K.T. Aust, U. Erb, P.C. Lichtenberger. GRAIN-BOUNDARY DESIGN AND CONTROL FOR INTERGRANULAR STRESS-CORROSION RESISTANCE, Scr. Metall. Materialia 25 (1991) 1775-1780.

[21] T. Watanabe. Grain boundary engineering: historical perspective and future prospects, J Mater Sci 46 (2011) 4095-4115.

[22] D.B. Bober, M. Kumar, T.J. Rupert. Nanocrystalline grain boundary engineering: Increasing Sigma 3 boundary fraction in pure Ni with thermomechanical treatments, Acta Mater 86 (2015) 43-54.

[23] K.N. Tu. Recent advances on electromigration in very-large-scale-integration of interconnects, J Appl Phys 94 (2003) 5451-5473.

[24] F. Bachmann, R. Hielscher, H. Schaeben. Texture Analysis with MTEX - Free and Open Source Software Toolbox, Texture and Anisotropy of Polycrystals Iii 160 (2010) 63-68. [25] G.S. Rohrer, J. Li, S. Lee, A.D. Rollett, M. Groeber, M.D. Uchic. Deriving grain boundary character distributions and relative grain boundary energies from three-dimensional EBSD data, Mater Sci Tech-Lond 26 (2010) 661-669.

[26] A.D. Darbal, K.J. Ganesh, X. Liu, S.B. Lee, J. Ledonne, T. Sun, B. Yao, A.P. Warren, G.S. Rohrer, A.D. Rollett, P.J. Ferreira, K.R. Coffey, K. Barmak. Grain Boundary Character Distribution of Nanocrystalline Cu Thin Films Using Stereological Analysis of Transmission Electron Microscope Orientation Maps, Microsc Microanal 19 (2013) 111-119. 
[27] X. Liu, N.T. Nuhfer, A.P. Warren, K.R. Coffey, G.S. Rohrer, K. Barmak. Grain size dependence of the twin length fraction in nanocrystalline $\mathrm{Cu}$ thin films via transmission electron microscopy based orientation mapping, J Mater Res 30 (2015) 528-537.

[28] H. Grimmer, W. Bollmann, Warringt.Dh. Coincidence-Site Lattices and Complete Pattern-Shift Lattices in Cubic-Crystals, Acta Crystallogr A A 30 (1974) 197-207.

[29] D.G. Brandon. Structure of High-Angle Grain Boundaries, Acta Metall Mater 14 (1966) 1479-\&.

[30] A.H. King, S. Shekhar. What does it mean to be special? The significance and application of the Brandon criterion, J. Mater. Sci. 41 (2006) 7675-7682.

[31] H.A. Schafft. Thermal-Analysis of Electromigration Test Structures, Ieee T Electron Dev 34 (1987) 664-672.

[32] C.V. Thompson. Structure evolution during processing of polycrystalline films, Annu Rev Mater Sci 30 (2000) 159-190.

[33] R.A. Baragiola. Sputtering: survey of observations and derived principles, Philos T Roy Soc A 362 (2004) 29-53.

[34] J. Lindhard. Influence of Crystal Lattice on Motion of Energetic Charged Particles, Matemat-Fysis Meddel 34 (1965) 1-\&.

[35] S. Olliges, P. Gruber, A. Bardill, D. Ehrler, H.D. Carstanjen, R. Spolenak. Converting polycrystals into single crystals - Selective grain growth by high-energy ion bombardment, Acta Mater. 54 (2006) 5393-5399.

[36] M. Seita, R. Schaublin, M. Dobeli, R. Spolenak. Selective ion-induced grain growth: Thermal spike modeling and its experimental validation, Acta Mater. 61 (2013) 6171-6177.

[37] S. Datz, C. Erginsoy, Leibfrie.G, H.O. Lutz. Motion of Energetic Particles in Crystals, Ann Rev Nucl Sci 17 (1967) 129-+. 
[38] S.J. Park, D.P. Norton, V. Selvamanickam. Ion-beam texturing of uniaxially textured Ni films, Appl. Phys. Lett. 87 (2005).

[39] E.W. Dickson, D.W. Pashley. Double Positioning in Silver and Gold Layers Deposited on Mica, Philos Mag 7 (1962) 1315-1321.

[40] K. Meinel, M. Klaua, H. Bethge. On Twin and Stacking-Fault Formation during the Epitaxial Film Growth of Fcc Materials on (111) Substrates, Phys Status Solidi A 110 (1988) 189-196.

[41] V. Randle. Twinning-related grain boundary engineering, Acta Mater. 52 (2004) 4067-4081.

[42] C.A. Schuh, R.W. Minich, M. Kumar. Connectivity and percolation in simulated grain-boundary networks, Philos Mag 83 (2003) 711-726.

[43] C.A. Schuh, M. Kumar, W.E. King. Universal features of grain boundary networks in FCC materials, J Mater Sci 40 (2005) 847-852.

[44] F. Ruffino, M.G. Grimaldi, C. Bongiorno, F. Giannazzo, F. Roccaforte, V. Raineri, C. Spinella. Normal and abnormal grain growth in nanostructured gold film, J Appl Phys 105 (2009).

[45] R.W. Balluffi. Grain-Boundary Diffusion Mechanisms in Metals, Metall Trans A 13 (1982) 2069-2095.

[46] O.V. Kononenko, V.N. Matveev, D.P. Field. Electromigration properties of multigrain aluminum thin film conductors as influenced by grain boundary structure, $\mathrm{J}$ Mater Res 16 (2001) 2124-2129.

[47] J. Cho, C.V. Thompson. Grain-Size Dependence of Electromigration-Induced Failures in Narrow Interconnects, Appl. Phys. Lett. 54 (1989) 2577-2579.

[48] S. Kasap. Principles of electronic materials and devices. 3rd ed., McGraw-Hill, Boston, 2006. 
[49] J.R. Black. Electromigration - a Brief Survey and Some Recent Results, Ieee T Electron Dev Ed16 (1969) 338-347.

[50] M. Etzion, I.A. Blech, Y. Komem. Study of Conductive Gold Film Lifetime under High-Current Densities, J Appl Phys 46 (1975) 1455-1458.

[51] R.E. Hummel, H.J. Geier. Activation-Energy for Electrotransport in Thin Silver and Gold-Films, Thin Solid Films 25 (1975) 335-342.

[52] K.L. Tai, M. Ohring. Grain-Boundary Electromigration in Thin-Films .2. Tracer Measurements in Pure Au, J Appl Phys 48 (1977) 36-45.

[53] S. Kilgore, C. Gaw, H. Henry, D. Hill, T. Schroder. Electromigration of electroplated gold interconnects, Materials, Technology and Reliability of Advanced Interconnects-2005 863 (2005) 313-318.

[54] W. Rupp, U. Ermert, R. Sizmann. Self-Diffusion Measurements in Gold Single Crystals between 286 Degrees C and 412 Degrees C, Phys Status Solidi 33 (1969) 509-\&.

[55] J. Wang, N. Li, A. Misra. Structure and stability of Sigma 3 grain boundaries in face centered cubic metals, Philos Mag 93 (2013) 315-327.

[56] D.S. Gianola, B.G. Mendis, X.M. Cheng, K.J. Hemker. Grain-size stabilization by impurities and effect on stress-coupled grain growth in nanocrystalline Al thin films, Mat Sci Eng a-Struct 483-84 (2008) 637-640.

[57] M. Frary, C.A. Schuh. Percolation and statistical properties of low- and high-angle interface networks in polycrystalline ensembles, Phys. Rev. B 69 (2004).

[58] M. Frary, C.A. Schuh. Connectivity and percolation behaviour of grain boundary networks in three dimensions, Philos Mag 85 (2005) 1123-1143.

[59] B.D. Knowlton, J.J. Clement, C.V. Thompson. Simulation of the effects of grain structure and grain growth on electromigration and the reliability of interconnects, J Appl Phys 81 (1997) 6073-6080. 
[60] H. Ma, Y. Zou, A.S. Sologubenko, R. Spolenak. Copper thin films by ion beam assisted deposition: Strong texture, superior thermal stability and enhanced hardness, Acta Mater 98 (2015) 17-28.

[61] M. Seita, C.M. Pecnik, S. Frank, R. Spolenak. Direct evidence for stress-induced texture evolution and grain growth of silver thin films upon thermal treatment and self-ion bombardment, Acta Mater 58 (2010) 6513-6525.

[62] W.Z. Han, M.J. Demkowicz, E.G. Fu, Y.Q. Wang, A. Misra. Effect of grain boundary character on sink efficiency, Acta Mater 60 (2012) 6341-6351.

[63] Y.P. Kim, D.C. Kwon, H.M. Choi, Y.W. Park, S.I. Lee. The effect of residual tensile stress on electromigration lifetime of metal lines passivated by various oxides, Proceedings of the Ieee 2000 International Interconnect Technology Conference (2000) 205-207.

[64] Y.J. Park, C.V. Thompson. The effects of the stress dependence of atomic diffusivity on stress evolution due to electromigration, J Appl Phys 82 (1997) 4277-4281.

[65] J.J. Clement, C.V. Thompson. Modeling Electromigration-Induced Stress Evolution in Confined Metal Lines, J Appl Phys 78 (1995) 900-904.

[66] Y.M. Wang, A.F. Jankowski, A.V. Hamza. Strength and thermal stability of nanocrystalline gold alloys, Scripta Mater 57 (2007) 301-304.

[67] H. Tanimoto, S. Sakai, H. Mizubayashi. Mechanical property of high density nanocrystalline gold prepared by gas deposition method, Nanostruct Mater 12 (1999) 751 756.

[68] W. Zhou, J.L. Yang, Y. Li, F.H. Yang. Fracture properties of PECVD silicon nitride thin films by long rectangular memrane bulge test, 2008 3rd Ieee International Conference on Nano/Micro Engineered and Molecular Systems, Vols 1-3 (2008) 261-264. 


\section{Figure captions}

Fig. 1. Schematics, in-plane orientation EBSD maps, and (111) pole figures illustrating the film microstructure evolution during iGBE. (a) Initial (111) textured microstructure (reference). First iGBE cycle comprising SGM (b) and supplemental deposition (c). Both the channeling-oriented grains and those in twinned orientation are represented in green color in the schematics as well as in the EBSD maps. Upon supplemental deposition, new grains in twinned orientation nucleate, as evidenced by the six-fold symmetry in the corresponding pole figure. (d and e) Second iGBE cycle.

Fig. 2. Secondary electron and backscattered electron micrographs showing Au coverage in $50 \mathrm{~nm}$ thick films through the entire iGBE process.

Fig. 3. GB character distribution in the $50 \mathrm{~nm}$ thick microstructure templates.

Fig. 4. Evolution of TJ distribution in $50 \mathrm{~nm}$ thick films as a function of iGBE cycles.

Fig. 5. SEM cross-sections of as-produced $500 \mathrm{~nm}$ thick films. (a) reference, (b) $1 \times$ iGBE and (c) $2 \times$ iGBE films.

Fig. 6. (a) EBSD in-plane orientation maps and corresponding (111) pole figures of $500 \mathrm{~nm}$ thick reference, $1 \times \mathrm{iGBE}$, and $2 \times \mathrm{iGBE}$ films. (b) Corresponding GB character distribution in $500 \mathrm{~nm}$ thick films, showing a substantial increase in the number fractions of $\Sigma 1$ and $\Sigma 3$ boundaries upon iGBE cycles. 
Fig. 7. Evolution of TJ distribution in $500 \mathrm{~nm}$ thick films as a function of iGBE cycles.

Fig. 8. (a) Layout of the Au interconnect lines for EM test. (b) Arrhenius plots of the TTF versus $1 / T$. Each data point represents the average $T T F$ of 3 to 5 tested lines. (c-e) SEM cross-sections of EM tested $500 \mathrm{~nm}$ thick lines. (c) reference, (d) $1 \times$ iGBE, and (e) $2 \times$ iGBE. The micrographs are from regions near to the cathode end. The arrows indicate the EMinduced damage.

Fig. 9. EBSD in-plane orientation maps and corresponding (111) pole figures of the $2 \times \mathrm{iGBE}$ microstructure (a) before and (b) after a 3 hour thermal annealing at $400^{\circ} \mathrm{C}$.

Fig. 10. Representative SEM images of line failure at (a) cathode and (b) anode in the $500 \mathrm{~nm}$ thick reference sample after EM test.

Fig. 11. (a) Comparison of the low-diffusivity GB number fraction, $p$, in the tested microstructures. The ranges of the defined percolation thresholds for the 2-D microstructure with both fiber texture and twins are also presented. (b) Electric circuit models of the GB networks in microstructures with different $p$. (top) $p>p_{L}$, low-diffusivity boundaries percolate; (middle) $p_{H}<p<p_{L}$, neither low-diffusivity nor high-diffusivity boundaries percolate; (bottom) $p<p_{H}$, high-diffusivity boundaries percolate.

Fig. 12. Representative TEM micrographs of EM tested lines. (a-c) are bright-field (BF) micrographs taken from (a) reference, (b) $1 \times$ iGBE and (c) $2 \times$ iGBE sample. (d) is a highangle annular dark-field (HAADF) STEM micrograph taken from the $2 \times$ iGBE sample. The 
TEM lamellae are cut from regions near the cathode end by FIB. The red dashed lines indicate where the interface between the $50 \mathrm{~nm}$ thick microstructure template and the remainder $450 \mathrm{~nm}$ thick material.

\section{Table captions}

Table 1. Average grain size and the area fraction of channeling-, twinned- and randomlyoriented grains as well as non-indexed areas in the $50 \mathrm{~nm}$ and $500 \mathrm{~nm}$ thick reference, $1 \times$ iGBE, and $2 \times$ iGBE films, respectively.

Table 2. Calculated lattice and grain boundary diffusivities in Au from [23].

Table 3. Summary of the total number fraction of low-diffusivity GBs, $p$, in reference, $1 \times \mathrm{iGBE}$, and $2 \times \mathrm{iGBE}$. The simulated values of the percolation thresholds for the existence of high- $\left(p_{H}\right)$ and low-diffusivity GBs $\left(p_{L}\right)$ in 2-D microstructures with either fiber texture or twins are also listed. 

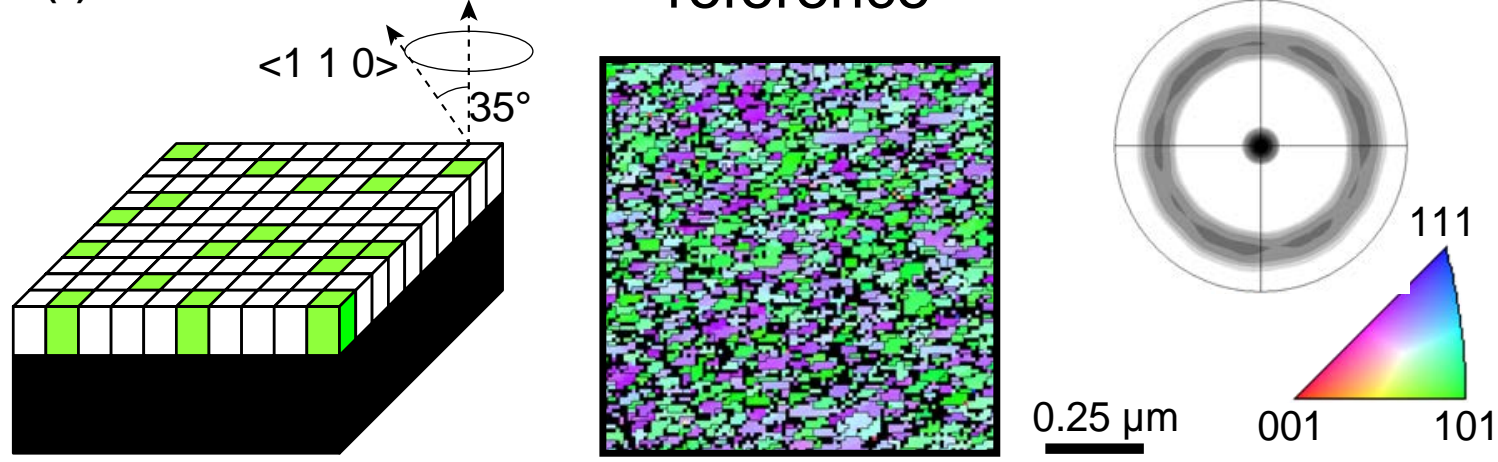

$1^{\text {st }}$ iGBE cycle <1 1 1>

b.
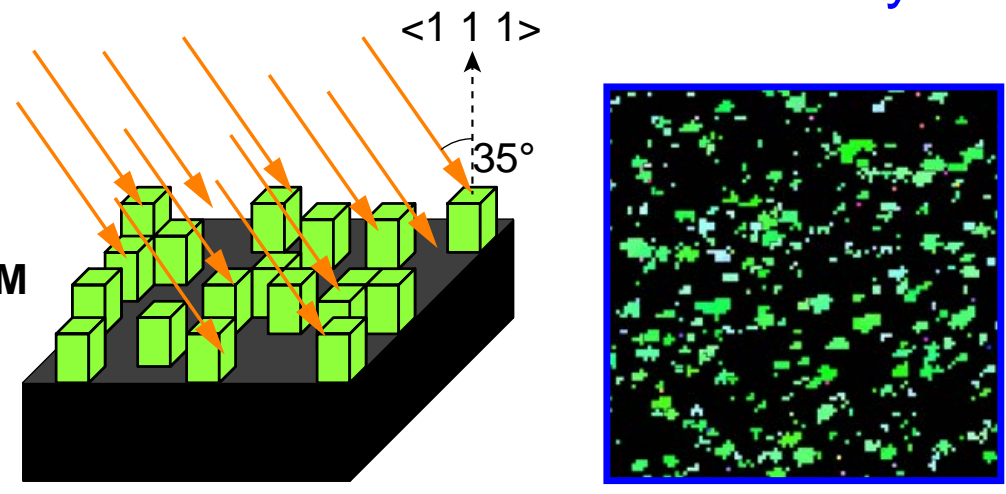

111

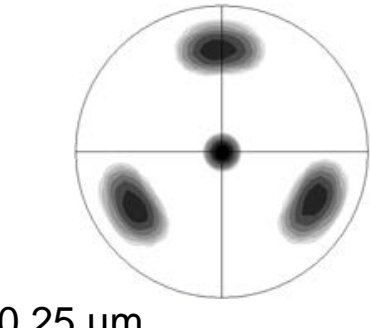

$0.25 \mu \mathrm{m}$

C.

Suppl.

Deposition

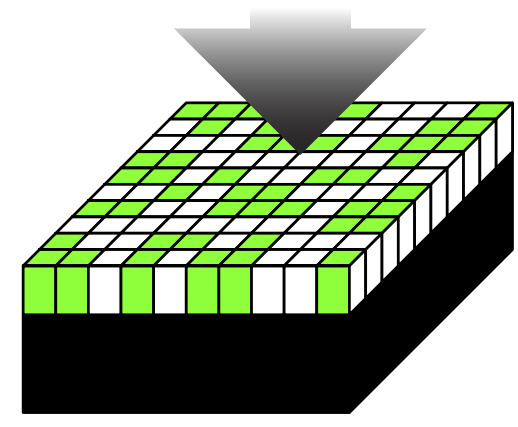

111

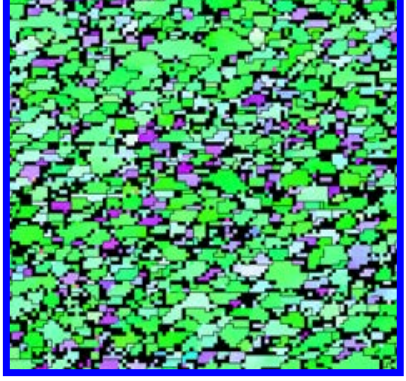

$0.25 \mu \mathrm{m}$ d <1 $11>$
SGM

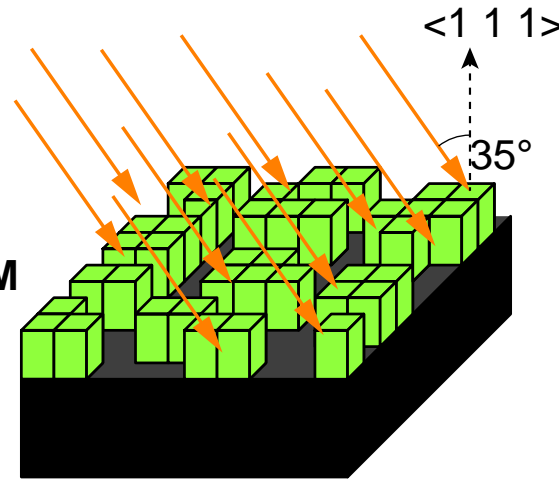

$2^{\text {nd }}$ iGBE cycle

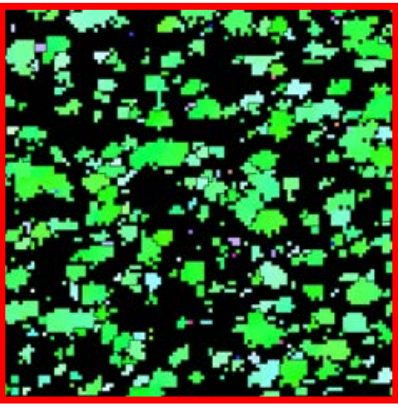

111

$0.25 \mu \mathrm{m}$ e

Suppl.

Deposition

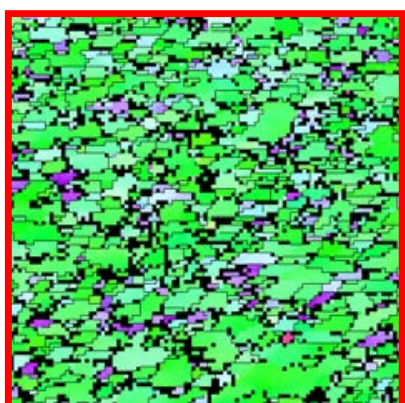

$0.25 \mu \mathrm{m}$ 
a.
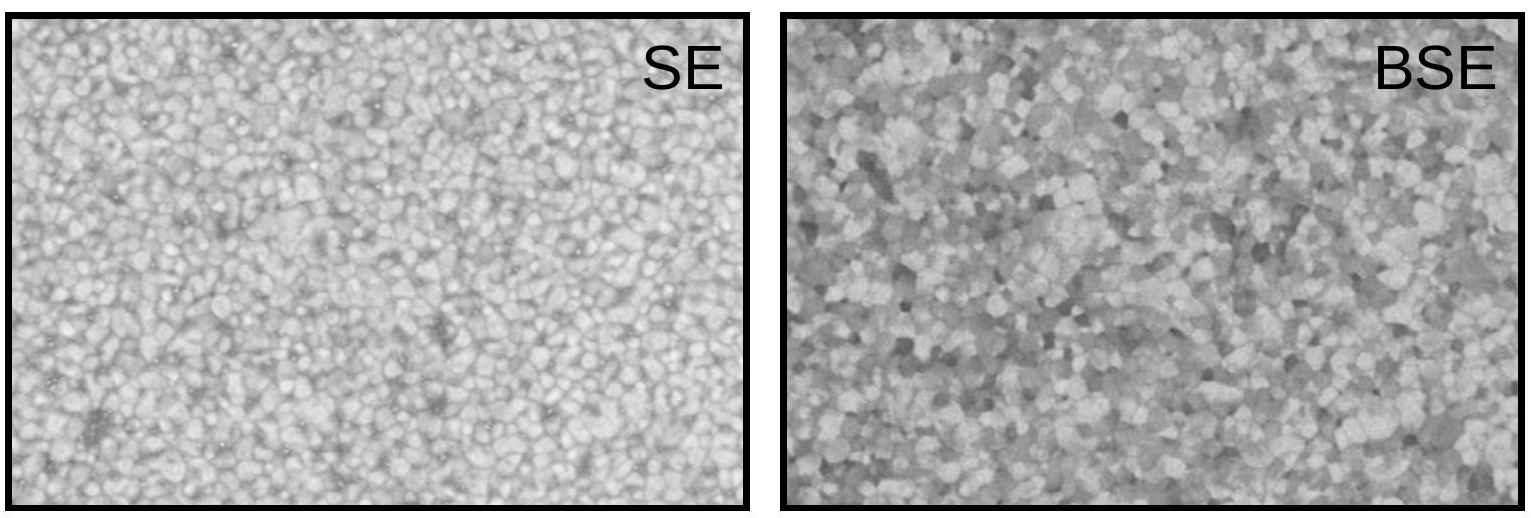

b.

$1^{\text {st }}$ iGBE cycle
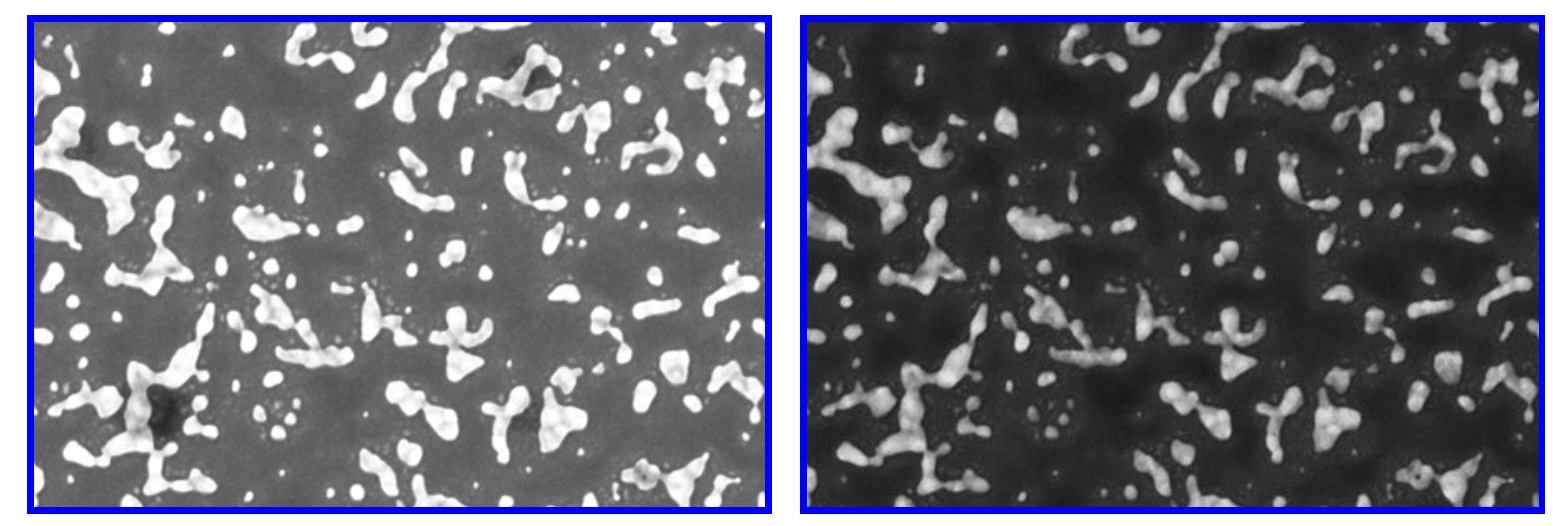

SGM

Suppl.

Deposition
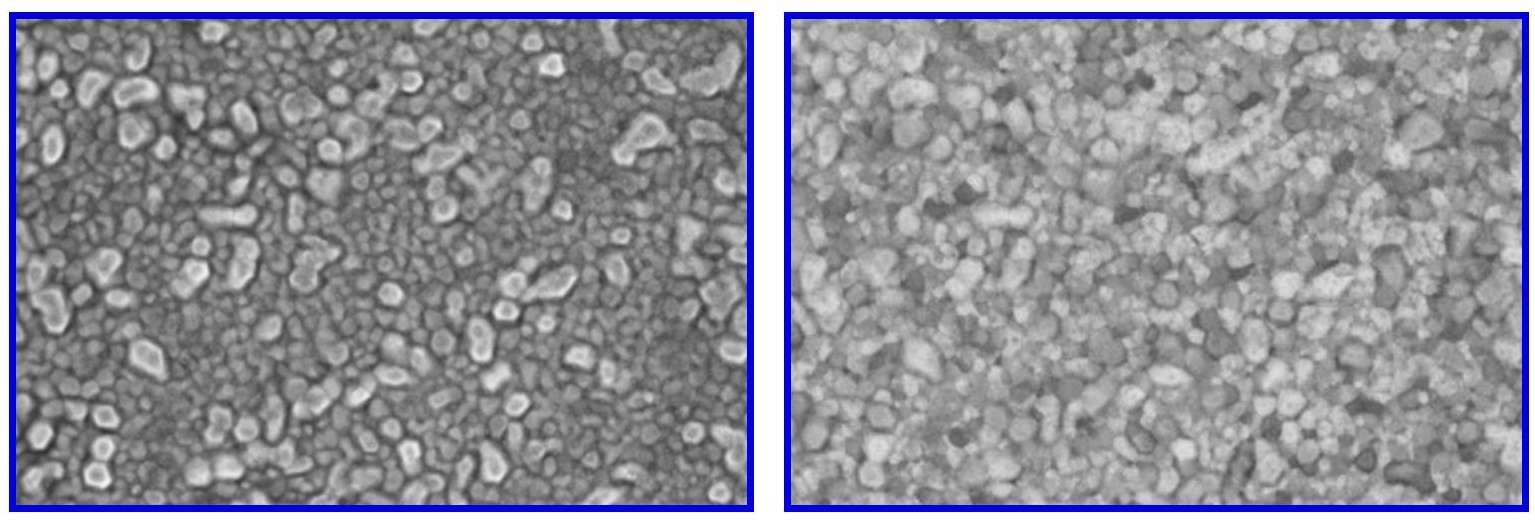

d.

$2^{\text {nd }}$ iGBE cycle

SGM

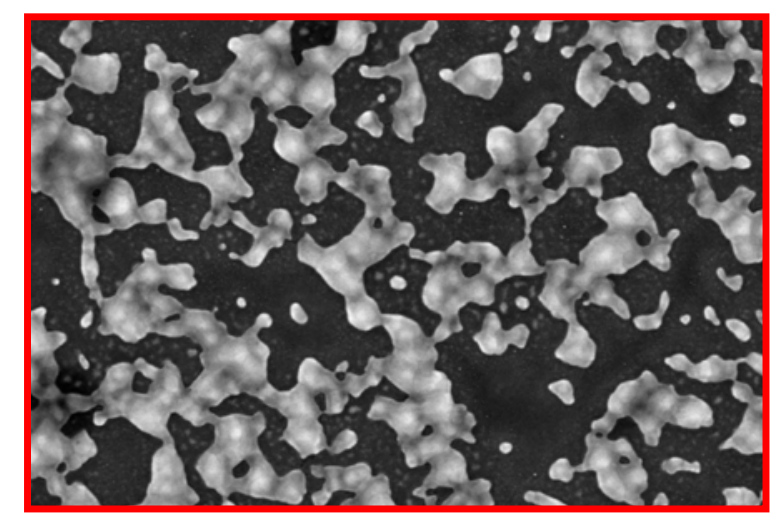

e.

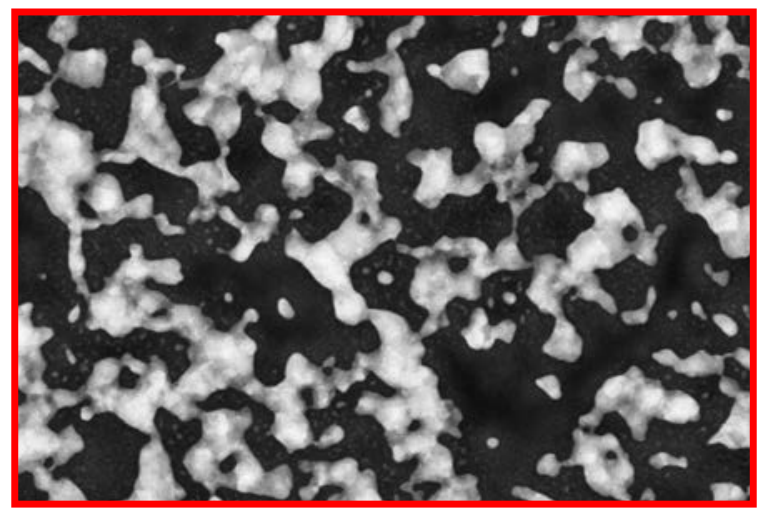

Suppl.

Deposition
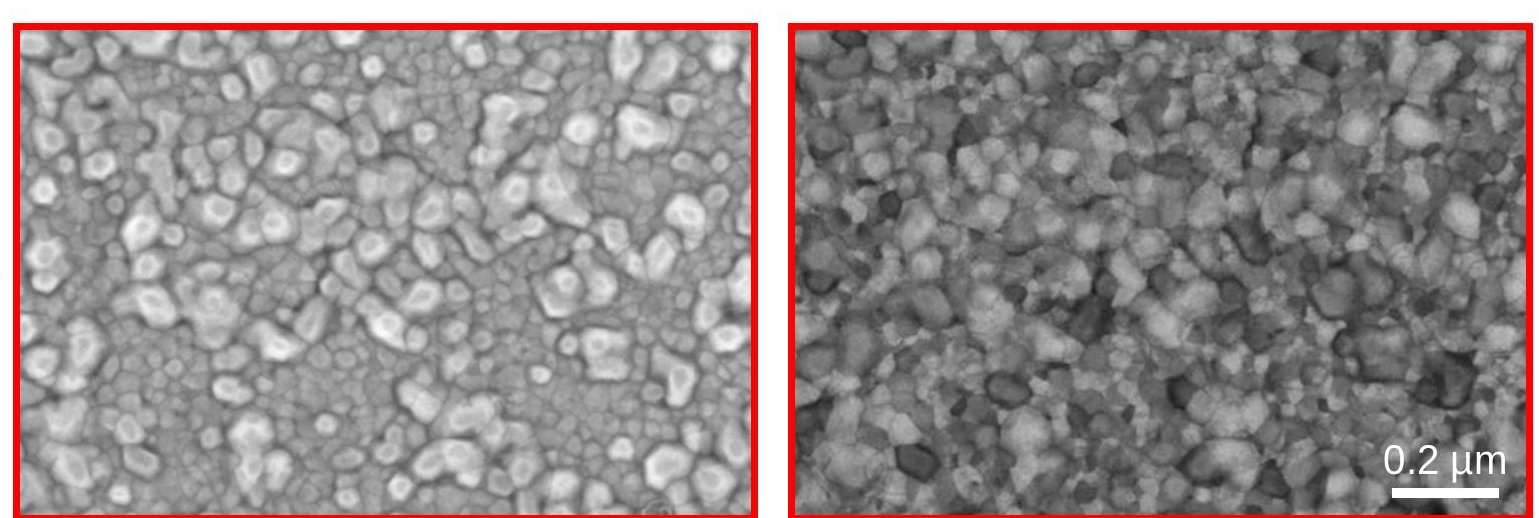


\section{Figuse(s)}

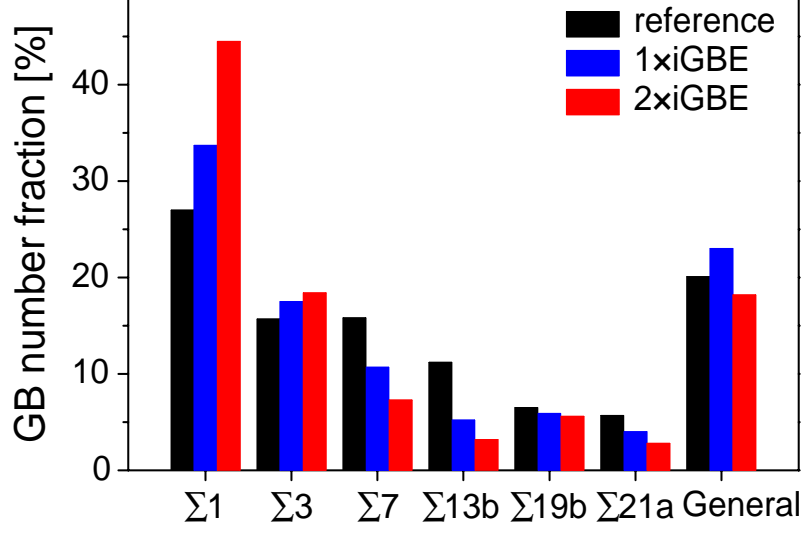


Figure(s)
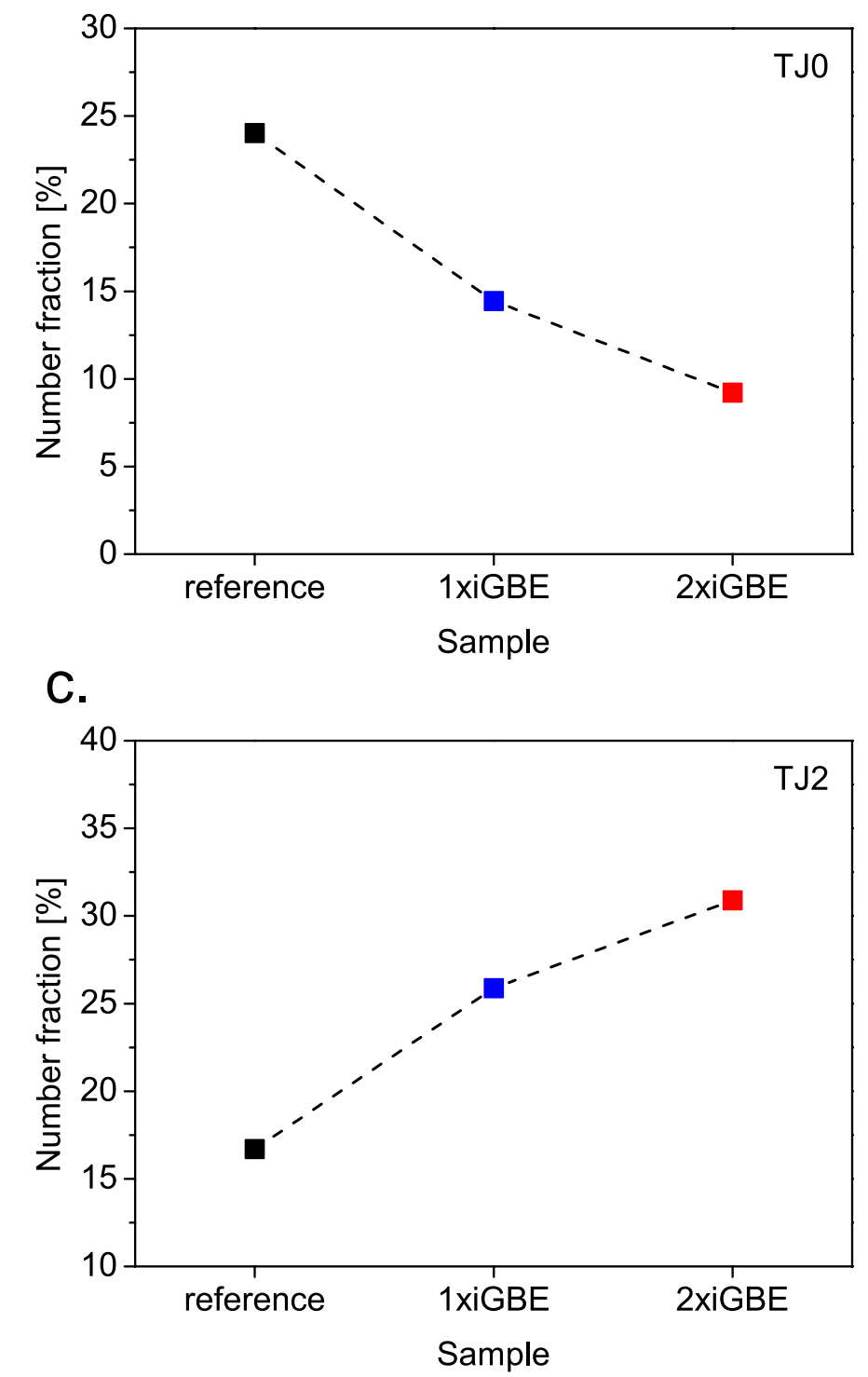

C. b.
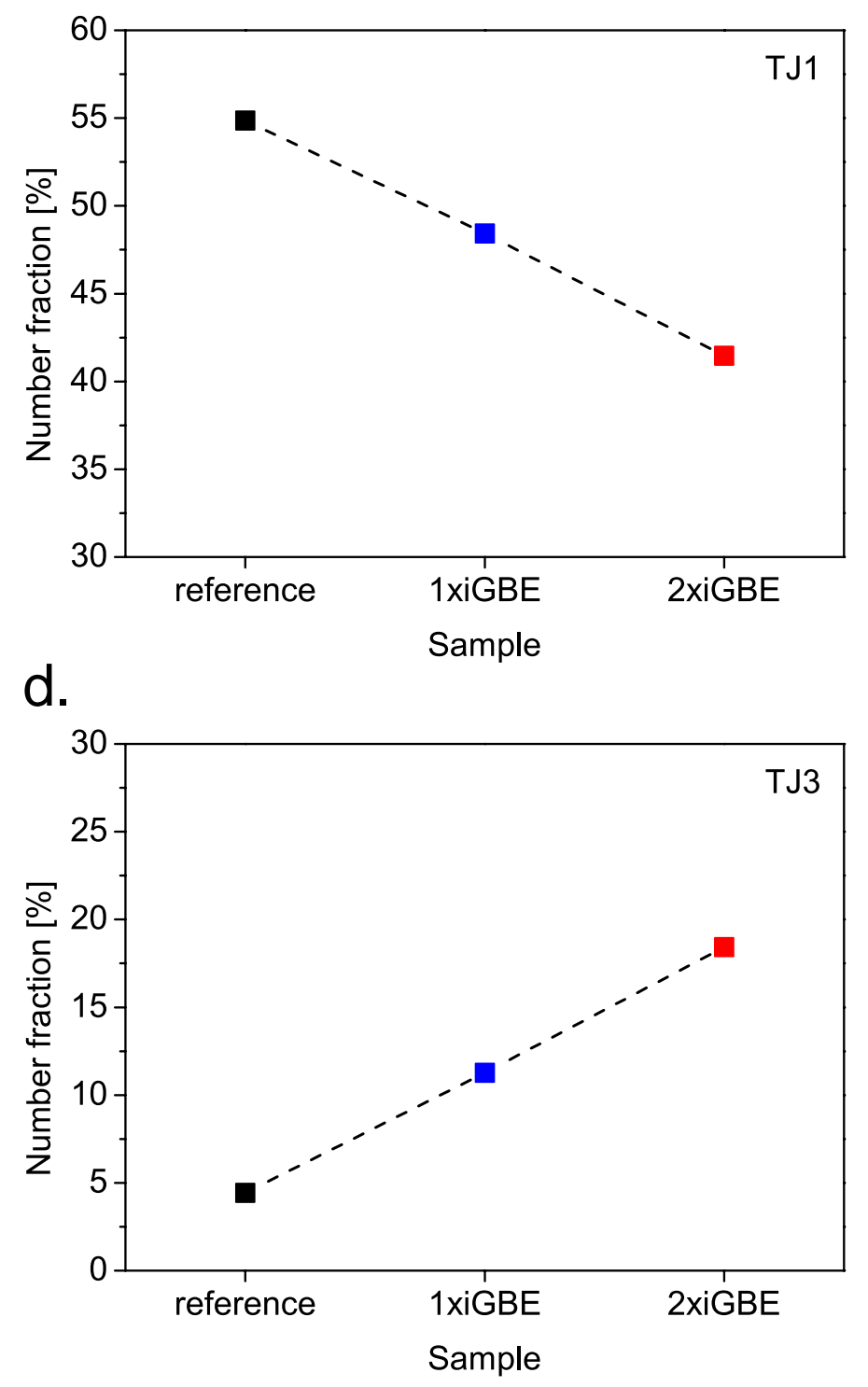
Figure(s)

reference

a.

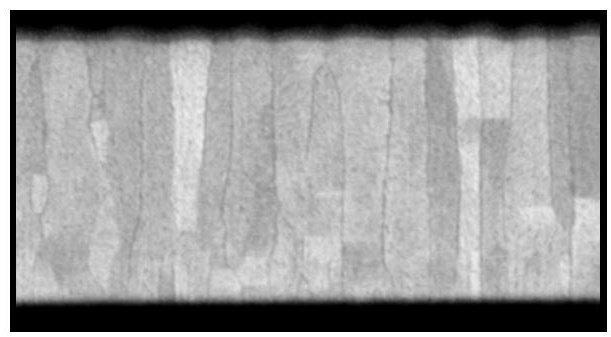

1×iGBE

b.

2×iGBE

C.

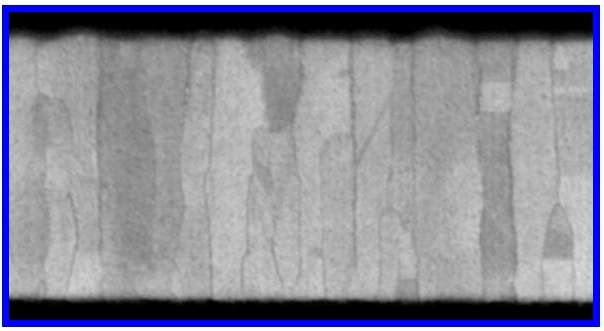

$0.2 \mu \mathrm{m}$

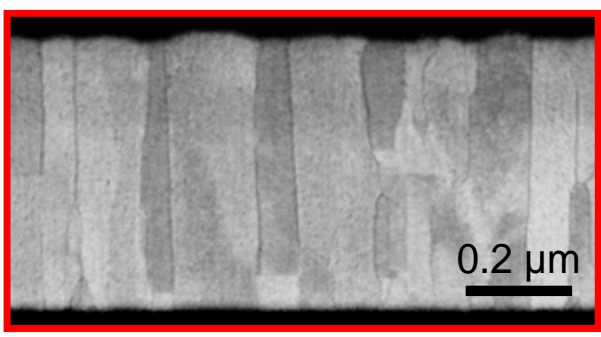


Figure(s)

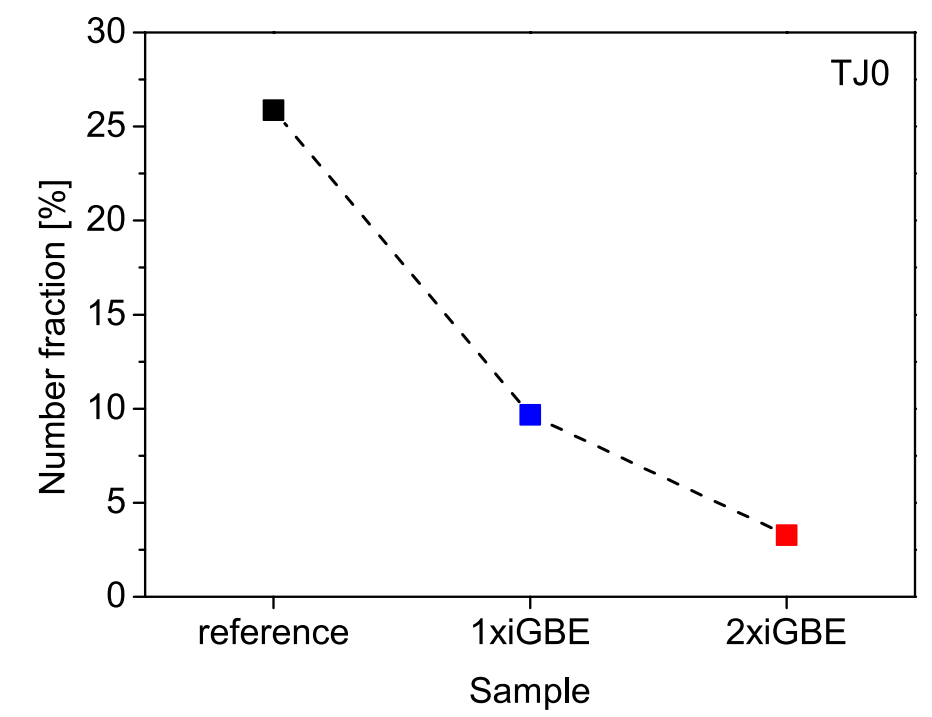

C.

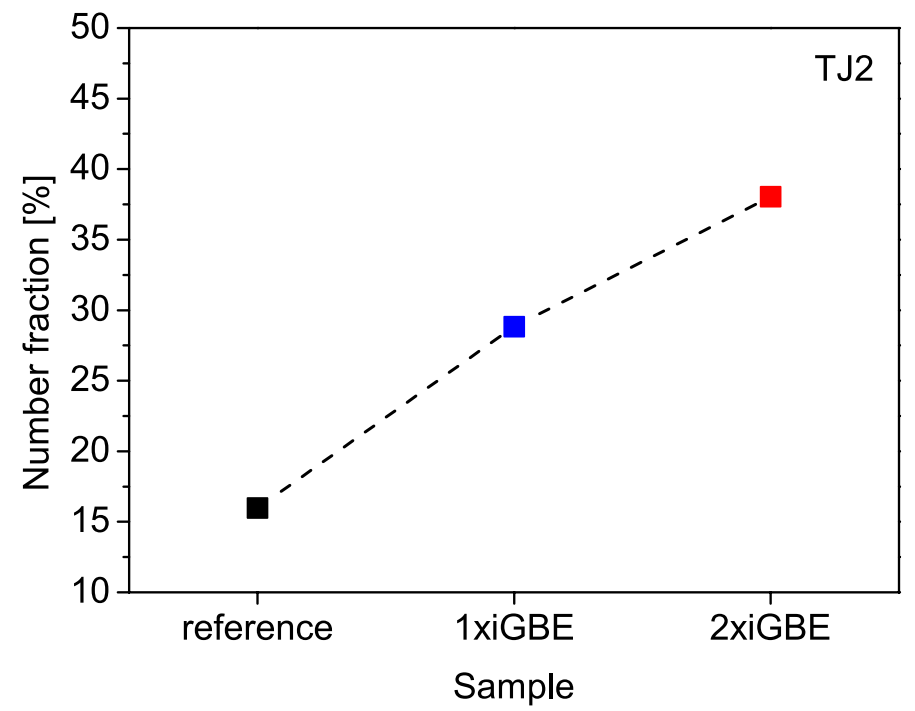

b.
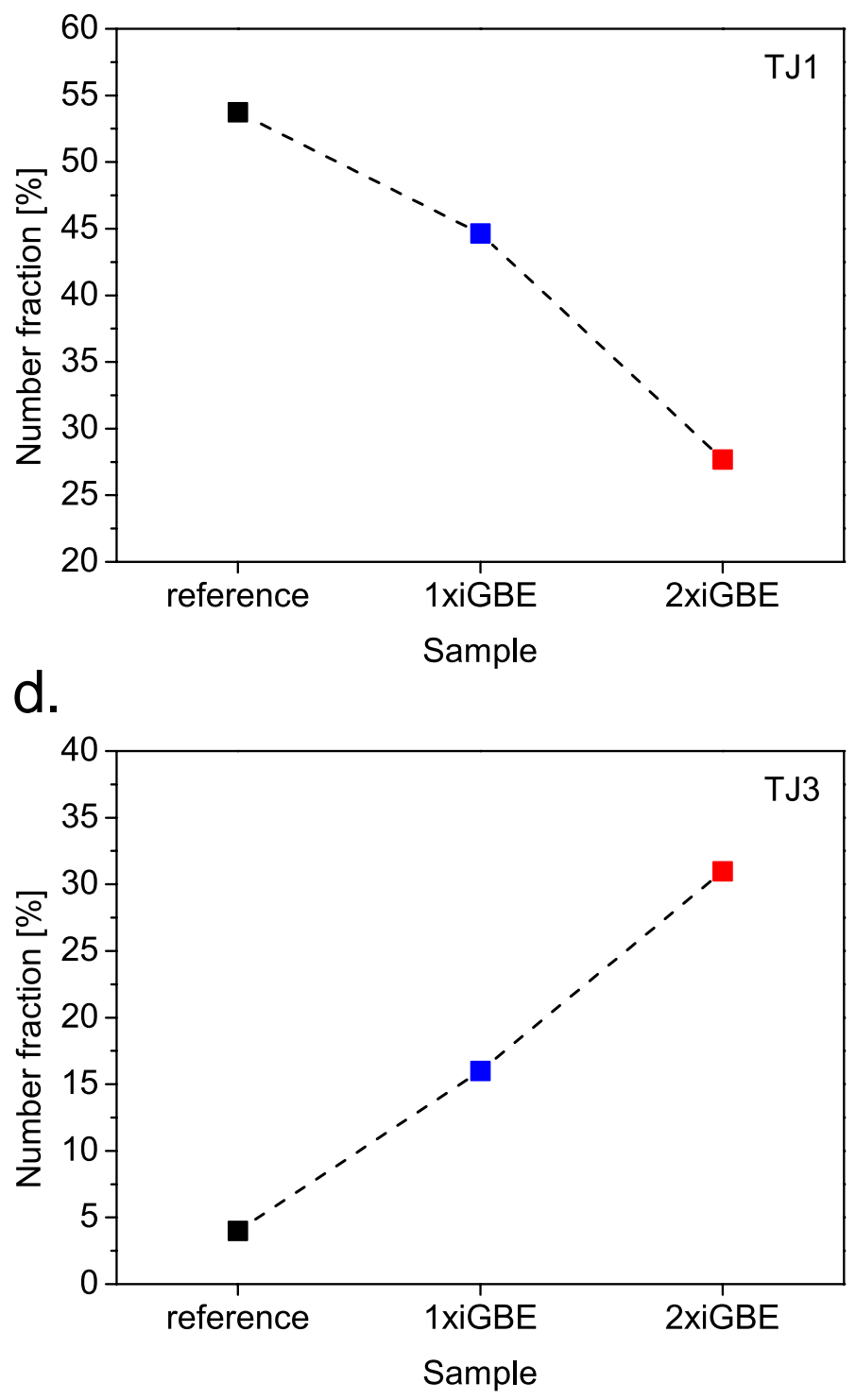
Figal. $\quad$ b.

\section{as-deposited \\ annealed}
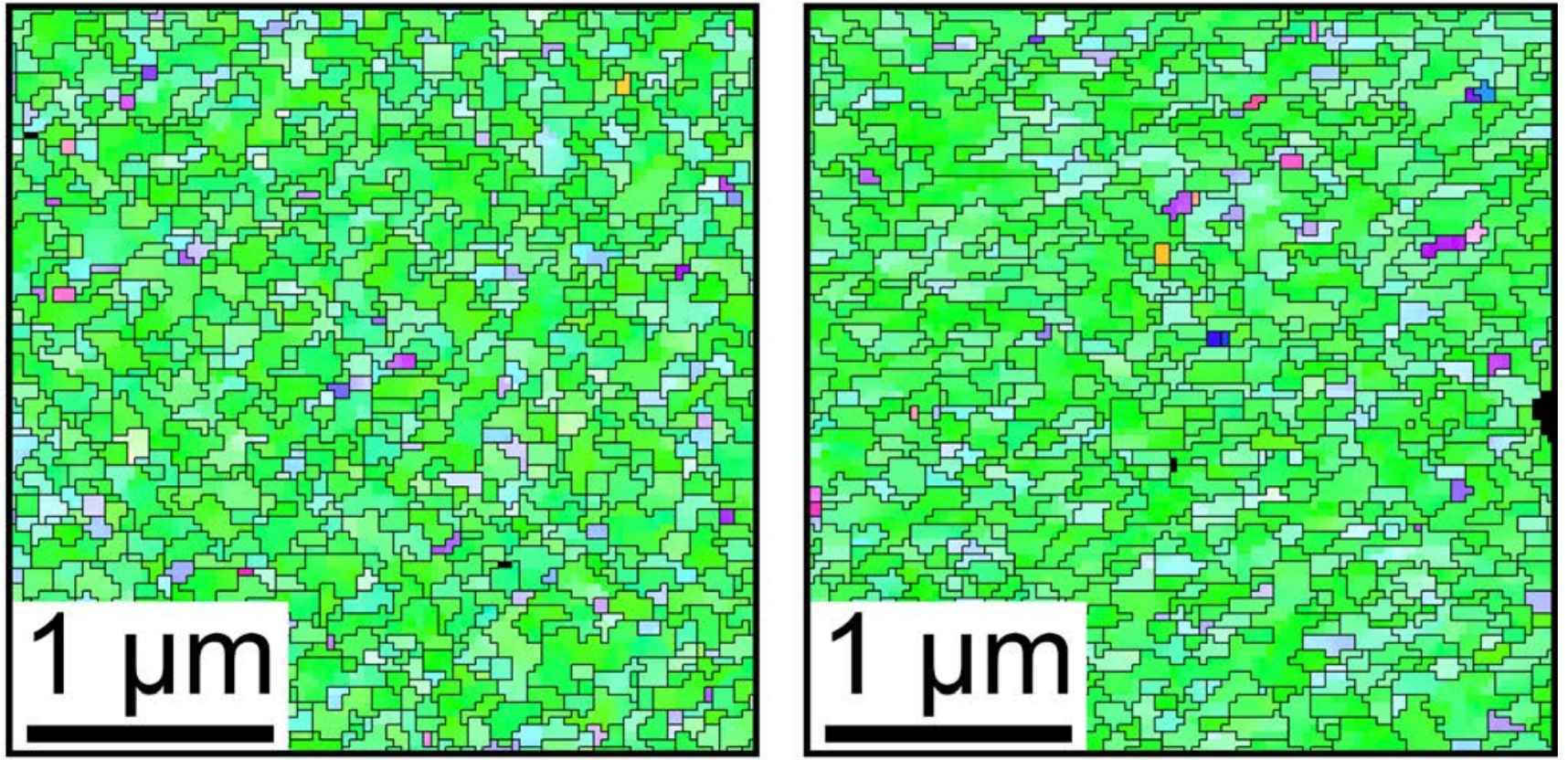

\section{1}

111
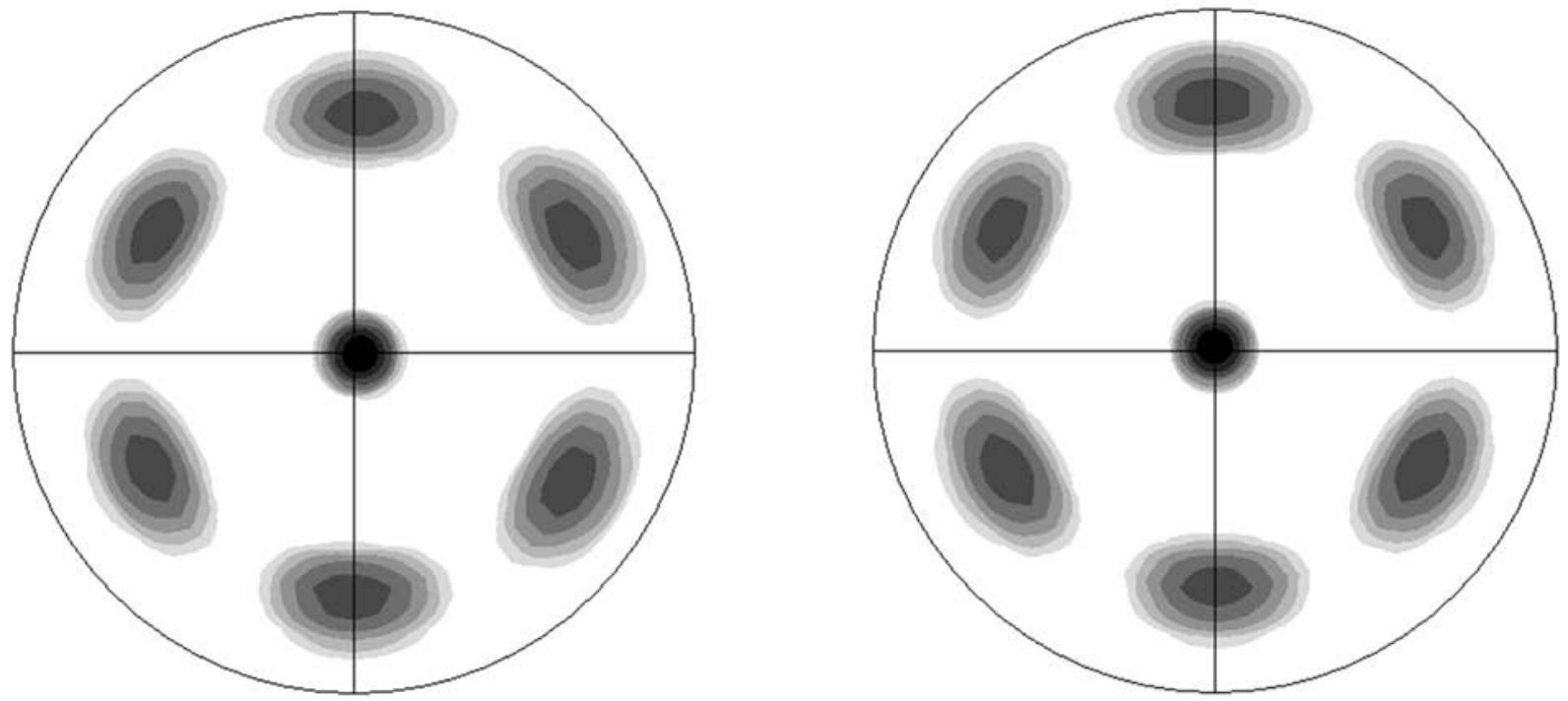
Figure(s) a.

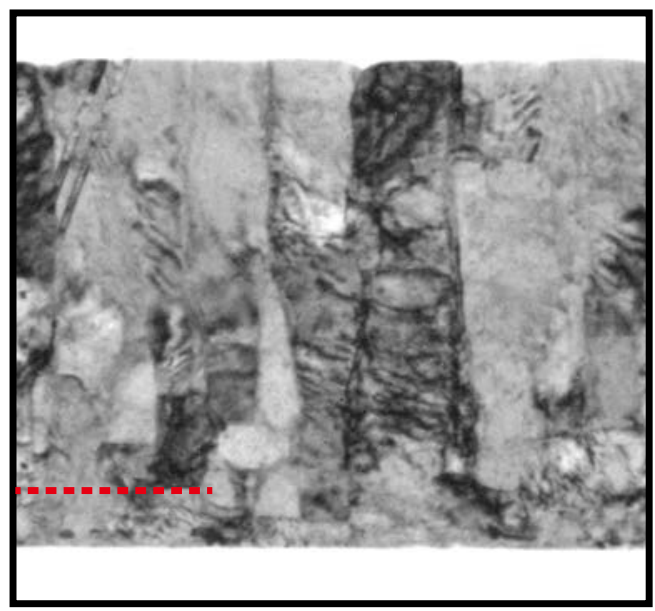

\section{2×iGBE}

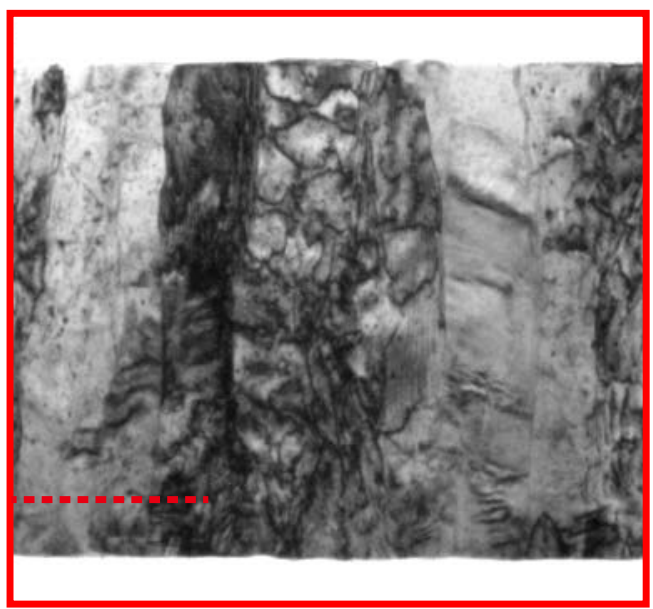

b.

1×iGBE

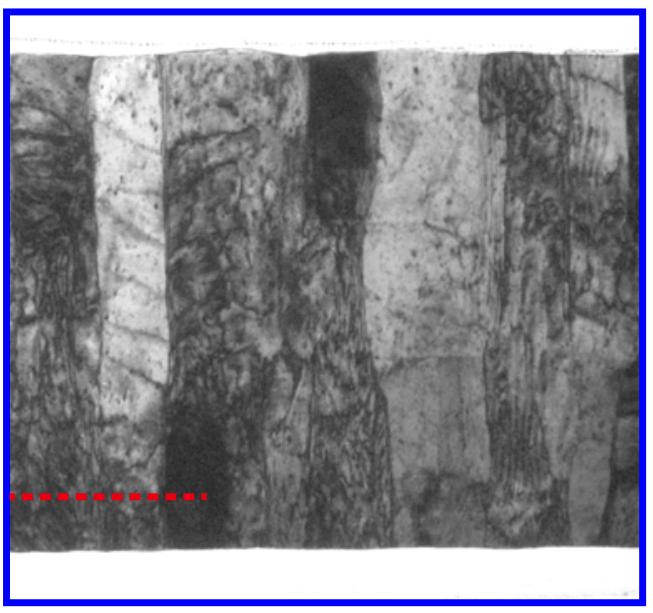

2xiGBE

$0.2 \mu \mathrm{m}$ 


\begin{tabular}{|c|c|c|c|c|c|c|}
\hline & Sample & $\begin{array}{l}\text { Grain size } \\
{[\mathrm{nm}]}\end{array}$ & $\begin{array}{l}\text { Channeling- } \\
\text { oriented grains }\end{array}$ & $\begin{array}{c}\text { Twinned- } \\
\text { oriented grains }\end{array}$ & $\begin{array}{c}\text { Randomly- } \\
\text { oriented grains }\end{array}$ & $\begin{array}{l}\text { Non-indexed } \\
\text { area }\end{array}$ \\
\hline \multirow{3}{*}{ 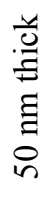 } & reference & & $25.7 \%$ & - & - & $31.9 \%$ \\
\hline & $1 \times \mathrm{iGBE}$ & $\sim 60$ & $39.7 \%$ & $26.4 \%$ & $6.3 \%$ & $27.6 \%$ \\
\hline & $2 \times \mathrm{iGBE}$ & & $48.7 \%$ & $22.2 \%$ & $1.7 \%$ & $27.4 \%$ \\
\hline \multirow{3}{*}{ 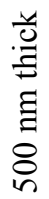 } & reference & & $32.8 \%$ & - & - & $9.5 \%$ \\
\hline & $1 \times \mathrm{iGBE}$ & $\sim 120$ & $45.3 \%$ & $43.1 \%$ & $4.2 \%$ & $7.4 \%$ \\
\hline & $2 \times$ iGBE & & $47.4 \%$ & $44.5 \%$ & $1.4 \%$ & $6.7 \%$ \\
\hline
\end{tabular}




\begin{tabular}{|c|c|c|c|}
\hline \multirow{2}{*}{$\mathrm{Au}$} & \multicolumn{3}{|c|}{ Diffusivity $\left[\mathrm{cm}^{2} / \mathrm{s}\right]$} \\
\hline & $100{ }^{\circ} \mathrm{C}$ & $200{ }^{\circ} \mathrm{C}$ & $300{ }^{\circ} \mathrm{C}$ \\
\hline Lattice & $1.2 \times 10^{-27}$ & $4.9 \times 10^{-22}$ & $2.3 \times 10^{-18}$ \\
\hline Grain boundary & $3.4 \times 10^{-15}$ & $3.0 \times 10^{-12}$ & $2.5 \times 10^{-10}$ \\
\hline
\end{tabular}




\begin{tabular}{|c|c|c|c|c|c|c|c|}
\hline \multicolumn{2}{|c|}{ Sample } & $\sum 1$ & $\sum 3$ & Others & $p$ & $p_{H}(2-\mathrm{D})$ & $p_{L}(2-\mathrm{D})$ \\
\hline \multirow{3}{*}{ 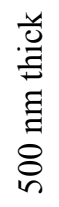 } & reference & $28.6 \%$ & $16.1 \%$ & $55.3 \%$ & $44.7 \% \pm 0.4 \%$ & - & - \\
\hline & $1 \times \mathrm{iGBE}$ & $35.3 \%$ & $23.6 \%$ & $41.1 \%$ & $58.9 \% \pm 1.2 \%$ & - & - \\
\hline & $2 \times \mathrm{iGBE}$ & $39.6 \%$ & $28.2 \%$ & $32.2 \%$ & $67.8 \% \pm 2.6 \%$ & - & - \\
\hline \multicolumn{2}{|c|}{ Fiber textured [57] } & - & - & - & - & $39.9 \%$ & $68.9 \%$ \\
\hline \multicolumn{2}{|c|}{ Twinned [58] } & - & - & - & - & $34.1 \%$ & $66.6 \%$ \\
\hline
\end{tabular}




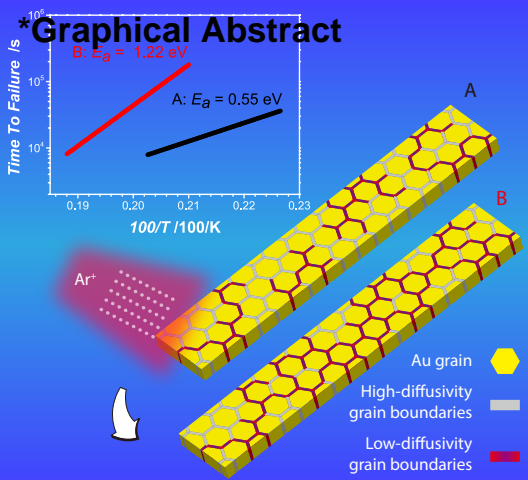

